\title{
THE EVOLUTION OF PLANETARY NEBULAE. III. POSITION-VELOCITY IMAGES OF BUTTERFLY-TYPE NEBULAE
}

\author{
VINCENT ICKE \\ Sterrewacht Leiden, Postbus 9513, 2300 RA Leiden, The Netherlands \\ Heather L. Preston ${ }^{a)}$ \\ Astronomy Program, Iowa State University, Ames, Iowa 50011 \\ BRUCE BALICK ${ }^{a)}$ \\ Astronomy Department, University of Washington, Seattle, Washington 98195 \\ Received 31 August 1988
}

\begin{abstract}
We present observations of the motions of the shells of the planetary nebulae NGC 2346, NGC 2371-2, NGC 2440, NGC 6058, NGC 6210, IC 1747, IC 5217, J-320, and M2-9. These are all "butterfly" type PNs, and show evidence for bipolar shocks. The observations are interpreted in terms of a fast spherical wind, driven by the central star into a quasitoroidal envelope deposited earlier by the star, during its slow-wind phase on the asymptotic giant branch. We show that this model, which is a straightforward extension of the mechanism we have invoked to account for elliptical PNs, reproduces the essential kinematic features of butterfly PNs. We infer that the envelopes of butterflies must have a considerable equator-to-pole density gradient, and suggest that the origin of this asphericity must be sought in an as yet unknown mechanism during the AGB, Mira, or OH/IR phases of late stellar evolution.
\end{abstract}

\section{INTRODUCTION}

A planetary nebula (PN) forms when a star of approximately solar mass reaches the end of its evolution on the asymptotic giant branch, and switches from losing mass in a fairly dense "slow wind" (usually identified with the Mira and $\mathrm{OH} / \mathrm{IR}$ stages of stellar evolution) to a mass-loss phase in which a tenuous "fast wind" blows the last traces of excess material off the stellar core, which remains as a young white dwarf ( see Balick, Preston, and Icke 1987, hereafter referred to as BPI1987, and references therein).

It was suggested by BPI1987 that planetary nebulae of the elliptical (E) morphological type (cf. Balick 1987, hereafter referred to as B1987) can be interpreted in terms of a single hydrodynamic model, namely one in which the red giant envelope (RGE), deposited by the central star of the PN while it was on the asymptotic giant branch, is globally inhomogeneous. If the RGE is roughly cylindrically symmetric and has a mild equatorial density excess, the shocks produced by the fast wind acquire geometries whose cross sections range from prolate spheroidal to plump figure-eight shapes (Kahn and West 1985; Icke 1988, hereafter referred to as I1988). Hydrodynamic focusing by the inner shock can produce cool and dense "soft bullets"' that propagate along the symmetry axis of the RGE, causing the characteristic "ansae" seen in many E type PNs (BPI1987; I1988; Soker and Livio 1988).

The existence of $\mathrm{E}$ type planetary nebulae, and the relative ease with which the BPI1987 hydrodynamical model reproduces the spectral observations, naturally leads one to suspect that other PN types may be likewise explained, given appropriate initial conditions. Good candidates might be older E types in which the elliptical outer shock has breached the confines of the RGE, or possibly younger ob-

a) Visiting Astronomer, Kitt Peak National Observatory, National Optical Astronomy Observatories (NOAO). The NOAO is operated by the Association of Universities for Research in Astronomy, Inc., under contract with the National Science Foundation (NSF). jects in which the equator-to-pole density contrast (henceforth called " $\mathrm{e} / \mathrm{p}$ ratio," meaning the density on the equator of the RGE divided by that on the symmetry axis, at a fixed distance from the central star) is larger than in the $E$ types. Indeed, candidates for both of these evolutionary stages have been found: they are members of the morphological class known as "butterfly" planetary nebulae (B1987).

This paper is a straightforward extension of BPI1987 towards these later evolutionary stages. Our goals are to (1) present detailed kinematic observations of butterfly PNs; (2) show the theoretically expected behavior of spectra and kinematic images of wind-driven shocks in inhomogeneous PN envelopes; (3) discover spectral-line features that are good diagnostics of aspherical shock propagation; (4) compare the synthetic spectra with observations, and draw conclusions about the RGE density distribution, PN age, and geometry.

\section{BIPOLAR KINEMATICS-MODERATE TO EXTREME}

\section{a) Observations}

The internal motions of some 45 planetary nebulae, in a sample chosen by B1987 and expanded by Balick and Preston (1987, hereafter referred to as BP1987), were studied in the lines of $\mathrm{H} \alpha$ and $[\mathrm{N} I \mathrm{I}] \lambda 6854 \AA$ (hereafter referred to as [N II] ). The kinematics of the PNs NGC 40, 2392, 3242, $6543,6826,7009,7354$, and 7662 have been fully described in BP1987 and BPI1987. In the present work, we use most of these data and include new data on the kinematics of the PNs NGC 2346, 2371-2, 2440, 6058, 6210; IC 1747 and 5217; $\mathrm{J}-320$, and M2-9. The data were obtained by use of the echelle spectrograph with CCD detector on the Mayall $4 \mathrm{~m}$ telescope of Kitt Peak National Observatory in April and December 1986. The collection and calibration of the data were carried out as described in BP1987, except where explicitly indicated. All velocities discussed in this paper are relative to the systemic velocity of the PN concerned. North, south, east, and west are abbreviated N, S, E, and W, respectively. 
The long slit of the echelle spectrograph was placed across one end of the image of each nebula as visible on the TV guider at the telescope. The first exposure was taken at the extreme $\mathrm{E}$ or $\mathrm{N}$ end (depending on whether the slit was oriented N-S or E-W). Prior to each subsequent exposure, the slit was uniformly offset perpendicular to the slit. All exposures in a series were taken with the same integration time. Details of the observations for the nebulae not discussed in BPI1987 are given in Table I. The slit was 1" wide for all nebulae except NGC 2346, 2371, and 6058, where a $1.5^{\prime \prime}$ slit was used. The seeing was about the same as the slit width, and the skies were generally photometric. The smoothing of the data $\left(8.91 \mathrm{~km} \mathrm{~s}^{-1}\right.$ in velocity and $1^{\prime \prime}$ along the slit) and comparison with instrumental resolution are as described in BPI1987. Some raw slit spectra are included to illustrate the correspondence with the synthetic spectra of the hydrodynamic models (Sec. III), and to provide the reader with a feeling for how the raw data relate to the final images.

The smoothed data for each planetary that had been mapped by the above process were packed into a three-dimensional data "cube" using the AIPS image-processing program, where the axes are right ascension, declination, and velocity. The data were then photographed as R.A.Dec. images, one such image for each $8.91 \mathrm{~km} \mathrm{~s}^{-1}$ interval along the velocity axis, and are shown in Figs. 1 and 2 [Plates 29-34]. These "isovelocity images" (also discussed in BPI1987) are presented as a series, from the extreme blue end of the velocity axis through the red end. These are followed by a single "summed image" which is the sum of the intensity distributions at all velocities. The final image in each figure of this type is the direct-CCD image taken at the KPNO $2.1 \mathrm{~m}$ telescope (B1987). It is included to give the reader a clear understanding of the geometrical distortion of the isovelocity images (this usually appears as a compression along the "offset" dimension, i.e., perpendicular to the slit).

It is important to note that the contrast of the figures that present the echelle data has been made nonlinear (though still monotonic) in order to show both subtle low-level features and much brighter highlights in a single frame. In spite of this, the full range of the data in the isovelocity images exceeds the dynamic range of the photographic medium used in this paper. Consequently, faint, extended features are sometimes no longer visible.

\section{b) Nebular Kinematic Features}

In this section, we describe the general morphological, kinematic, and other properties of interest for each nebula that are relevant to subsequent sections. The figure numbers

TABLE I. Description of kinematic images.

\begin{tabular}{lrrcc}
\hline \hline Nebula & $\begin{array}{c}\text { Slit } \\
\text { P.A. }\left({ }^{\circ}\right)\end{array}$ & $\begin{array}{c}\text { No. slit } \\
\text { positions }\end{array}$ & $\begin{array}{c}\text { Integ. time } \\
\text { per exposure }\end{array}$ & $\begin{array}{c}\text { Rel. slit } \\
\text { spacing (") }\end{array}$ \\
\hline NGC 2346 & 0 & 11 & 300 & 4 \\
NGC 2371-2 & 270 & 13 & 300 & 4 \\
NGC 2440 & 270 & 9 & 200 & 2 \\
NGC 6058 & 0 & 11 & 300 & 2 \\
NGC 6210 & 350 & 5 & - & -2 \\
IC 1747 & 0 & 7 & 400 & 2 \\
IC 5217 & 270 & 5 & 300 & 3 \\
J-320 & 270 & 7 & 800 & - \\
M2-9 & 0 & 4 & - & \\
\hline \hline
\end{tabular}

refer to the appropriate nebular kinematic images.

NGC 2346 (Fig. 1). Classified (B1987) as a "middle butterfly with open ends [displaying] moderate-to-high ionization," this nebula has a strong contrast in emission measure between the central region and the faint outer lobes. The central region has the appearance in $\mathrm{H} \alpha$ and [ $\mathrm{N} \mathrm{II}$ ] of a tilted ring of about $15^{\prime \prime}$ radius surrounding the central star. The inclination of the ring is $40^{\circ}$ if the ring is intrinsically circular.

A brightened disk (dust?) perpendicular to the bipolar lobes has been detected by speckle interferometry (Meaburn et al. 1985). Molecular hydrogen emission is seen surrounding the nebula and is especially bright near the perimeter of the central regions (Balick, Gatley, and Zuckerman 1988), much like CO (Healy and Huggins 1988 ). The central star is less affected by extinction than nearby nebulosity, suggesting that the line of sight to the star does not intercept the dense disk. Dust lanes are seen a few seconds north of the central star in $\mathrm{H} \alpha$ and [N II] CCD images (B1987), in apparent agreement with this interpretation.

The central star is a main-sequence A star with, presumably, an undetected companion which ionizes the gas. Several years ago the system began eclipsing with a 17 day period; however, the amplitude of the eclipses is decreasing, and the eclipsing object is now thought to be a compact, dissipating dust cloud (see Costero et al. 1986 for a discussion). The mass of the disk and the nearby molecular clouds is about $10 \%$ or less of the mass of the central star(s) (Healy and Huggins 1988).

The kinematics of the ionized regions has been discussed by Walsh (1983), and that of the neutral gas by Healy and Huggins (1988). The present data, which cover the nebula much more systematically than either earlier study, show the systematic motions of the nebula to be more complex than was hitherto known. Even so, there is a pattern to the kinematics which, on first inspection, seems consistent with the idea that the nebular lobes consist of gas flowing outward from the central regions of NGC 2346 where the central disk and stars are situated. The grand pattern of motion is somewhat obscured by the patchiness of the nebular emission and the tilt of the nebula with respect to the line of sight.

The isovelocity images show that the central, bright region of the nebula is visible to $\pm 25 \mathrm{~km} \mathrm{~s}^{-1}$. The best source of information about the kinematics of the lobes is the original set of spectrograms, one of which is reproduced in Fig. 3. The original spectra of NGC 2346 show extremely narrow lines at positions corresponding to the faint lobes, while the portion of each spectral line arising from the inner nebular regions near the star and the inclined disk appears as a tilted elliptical cavity. As will be shown below, the tilt of the spectral line is not necessarily a result of nebular rotation, but rather a "false rotation" phenomenon caused by an $\mathrm{e} / \mathrm{p}$ ratio on the order of 1.2 and an inclination angle between $30^{\circ}$ and $40^{\circ}$.

NGC 2371-2 (Fig. 2(a)). The morphological designation is "late elliptical." Only the $\mathrm{H} \alpha$ image is displayed owing to the low signal-to-noise ratio of the [ $\mathrm{N}$ II] data. The faint bipolar outer halo visible in the direct-CCD images is too faint to be detected using the echelle/CCD system.

NGC 2371-2 is a "moderate-to-high ionization" nebula (B1987). Its wind luminosity is close to the integrated nebular luminosity (Cerruti-Sola and Perinotto 1985), which suggests that much of the nebular energy is supplied by the wind and, therefore, wind shaping has probably been par- 
ticularly important for this object. Faint ansae NNE and SSW of the central cavity appear in the images, especially near zero velocity.

The detectable $\mathrm{H} \alpha$ emission spans a velocity range of $\pm 36 \mathrm{~km} \mathrm{~s}^{-1}$. The two brighter emission knots diametrically $\mathrm{N}$ and $\mathrm{S}$ of the central star are displaced in velocity by about $\pm 10 \mathrm{~km} \mathrm{~s}^{-1}$ from the systemic nebular velocity. Fainter regions $\mathrm{E}$ and $\mathrm{W}$ of the central star have relative motions about twice as large as those of the N-S knots. This pattern of motions is also seen in IC 1747 (although somewhat more extreme in velocity offset), and is not compatible with any simple pattern of uniform rotation.

NGC 2440 (Fig. 2(b)). The [N II] line is brighter than $\mathrm{H} \alpha$ (the ratio $[\mathrm{N} \mathrm{II}] / \mathrm{H} \alpha$ increases from about three to 30 from the central to the outer zones of the nebula). For this reason, and because the [N $\mathrm{NI}$ ] line is far less affected by thermal broadening than is $\mathrm{H} \alpha$, we present only the [N II] kinematic image. The slit of the echelle spectrograph was only stepped across the inner regions of the nebula, owing to the brief period in which NGC 2440 is observable from the northern hemisphere. Note that $\mathrm{E}$ is at the top and $\mathrm{S}$ is to the left in Fig. 2(b).

This bipolar "late butterfly" shows both extremely bright lines of low ionization such as [N II] and high ionization such as He II $\lambda 4686$. Studies of the variations in line ratios are under way in this object and will be reported elsewhere. $I U E$ spectra show that the central star is one of the hottest stars ever observed (Heap 1987; see Reay, Walton, and Atherton (1988) for a summary of the observations of this object).

The most remarkable kinematics of NGC 2440 were explored by Heathcoate and Weller (1987) and others. The present data are the first to systematically cover the nebula and to display the results as isovelocity images. The present kinematic images suggest that there are two independent pairs of lobes. The larger pair consists of nearly round bubbles nearly $\mathrm{E}$ and $\mathrm{W}$ of the central star. Each lobe spans about ten isovelocity images $\left(90 \mathrm{~km} \mathrm{~s}^{-1}\right)$, and the systemic velocities of the lobes are offset in velocity by about 70 $\mathrm{km} \mathrm{s}^{-1}$. Inside the large outer pair of lobes is a second, smaller, and much more elliptical pair in P.A. $-45^{\circ}$. Emission from the inner pair of lobes spans about $50 \mathrm{~km} \mathrm{~s}^{-1}$ in Fig. 2(b).

NGC 6058 (Fig. 2(c)). This nebula is not treated in B1987, but its resemblance to NGC 6720 suggests a similar morphological classification: middle elliptical, possibly with a high inclination angle. This would agree with the spectral line shapes, which give evidence of the "false rotation" phenomenon typical of that morphological class.

NGC 6210 (Fig. 2(d)). Morphologically, this nebula is puzzling; it is classified as an irregular, and it appears in $\mathrm{H} \alpha$ and radio images as a small, bright rectangle. In the CCD images of B1987, the rectangle is seen to break up into about four small knots in lines of low ionization such as [N II]. There is a very faint pair of outer knots (or perhaps ansae) about $20^{\prime \prime}$ $\mathrm{N}$ and $\mathrm{S}$ of the central region. Note that a streak of faint emission in the lower region of the fifth [N $\mathrm{NI}$ ] isovelocity image is a CCD defect.

Much as the $\mathrm{H} \alpha$ image is amorphous, the $\mathrm{H} \alpha$ line profile is broad (FWHM $40 \mathrm{~km} \mathrm{~s}^{-1}$ ) but otherwise unstructured. By contrast, the [ $\mathrm{N}$ II] line shows that the E regions of the main body of the nebula have large negative velocities, while the opposite is true for the W regions. Indeed, the NE and SW knots have about equal and opposite velocities, as does the
NW and SE pair. This is roughly the pattern of the motions seen in NGC 2371-2 and IC 1747, except that the intensities of the various knots are highly disparate in the case of NGC 6210. One can also view the system as containing expansion and rotation motions of order $50 \mathrm{~km} \mathrm{~s}^{-1}$. Both interpretations are somewhat ad hoc. A model for the motions and structure of NGC 6210 is obviously fraught with difficulties.

IC 1747 (Fig. 2(e)). This PN appears at first to have a nearly circular geometry; however, it is suspected (B1987) of being an irregular or middle elliptical with possibly large bipolar lobes camouflaged by their high degree of inclination. The isovelocity images show the $\mathrm{S}$ region of the nebula to be approaching and the $N$ lobe to be receding at \pm 53 $\mathrm{km} \mathrm{s}^{-1}$, reinforcing the impression of a high inclination angle. The raw spectral line shapes suggest this as well by exhibiting false rotation.

IC 5217 (Fig. 2(f)). This is an "early butterfly with open ends, possessing a sharp ionization front only in selected portions of its boundary (therefore only partly ionization bounded)" (B1987). It strongly resembles the Red Rectangle. Note that $S$ is at the left and $E$ is at the top in Fig. 2(f).

In the [N II] line, the bright linear feature appears to have the kinematics of a ring (or, perhaps, a disk with a central hole) expanding at $25 \mathrm{~km} \mathrm{~s}^{-1}$ and seen nearly edge-on. There is no hint of any systematic rotation. The $\mathrm{H} \alpha$ line neither confirms nor refutes this interpretation of the kinematics, owing to the effects of thermal broadening. The very faint lobes are not detected in these high-dispersion observations.

J-320 (Fig. 2(g)). Exhibiting an elongation accentuated by the presence of faint ansae at both ends, this PN has been classified (B1987) as either "peculiar or early butterfly." J320 is of particular interest in either case. It is small in angular size, with a radius near 3.2" (distance approximately 4 kpc-Acker et al. 1982). The slit width is thus relatively large in projected physical dimensions. The central star has strong P Cygni profiles in the lines of $\mathrm{N}$ III and $\mathrm{O}$ V (Perinotto 1983).

J-320, like NGC 6210, is spatially complex. Aside from the central body of the nebula there are two pairs of ansae. One pair is $\pm 12^{\prime \prime} \mathrm{N}$ and $\mathrm{S}$ of the nebular center. The inner pair is about $\pm 10^{\prime \prime}$ in P.A. $330^{\circ}$. Neither pair lies close to a symmetry axis of the inner region.

The kinematics are equally enigmatic. Each ansa of a pair has equal and opposite velocity to its counterpart (the inner pair has the largest velocity separation; however, this could be merely a projection effect). However, no overall pattern of motion is evident, which is also similar to NGC 6210 .

M2-9 (Fig. 2(h)). By far the most interesting butterfly morphologically, M2-9 presents many kinematic puzzles. B1987 classifies M2-9 as "butterfly with open ends." The morphology includes multiple sets of ansae at the edges of the inner regions. Strangely, however, pairs of knots show reflection symmetry about the (approximately $\mathrm{E}-\mathrm{W}$ ) equatorial line rather than inversion symmetry through the central star. These knots have extremely large E-W proper motions (Kohoutek and Surdej 1980). Balick (1988) remarks that the nucleus of M2-9 is similar to that of a slow nova or symbiotic star. Thus, M2-9 may not be a bona fide PN; nevertheless, it is an interesting hydrodynamical system which merits full consideration here.

Figure 2(h) shows faint $\mathrm{H} \alpha$ emission $\mathrm{N}$ and $\mathrm{S}$ of the central star extending over at least ten kinematic frames. The wavelength behavior of this emission is much like that of the 
circumstellar $\mathrm{H} \alpha$ emission line. Hence we suspect that the broad nebular $\mathrm{H} \alpha$ emission is actually stellar light scattered into the line of sight of the lobes.

The $\mathrm{S}$ and $\mathrm{N}$ lobes are shifted to the blue and the red with respect to the velocity of the central star by about \pm 45 $\mathrm{km} \mathrm{s}^{-1}$. Since it appears that the nebula has a very high inclination angle, the true space velocities of the lobes are certain to be far higher. As we shall argue in subsequent sections, the extreme elongation and narrow waist of this PN imply an unusually high $\mathrm{e} / \mathrm{p}$ ratio, with an axial tilt of about $15^{\circ}$.

\section{THEORETICAL POSITION-VELOCITY IMAGES}

\section{a) Behavior of the Shock Fronts}

We will now investigate whether the model presented by BPI1987 for elliptical PNs also applies to butterfly nebulae. Our hypothesis is that butterfly PNs are in all respects similar to ellipticals, except that the RGEs of butterflies are much more aspherical than the envelopes that surround the E types. Thus, we use the same analysis of shock motion, but with RGE parameters that are suitably more extreme.

Following I1988, we assume that the propagation of the shock that moves into the RGE is governed by one parameter only, namely the ratio of the pressure $P_{1}$ behind the shock and the RGE density $\rho_{0}$ ahead of it:

$$
K \equiv \frac{\gamma+1}{2} \frac{P_{1}}{\rho_{0}}
$$

Here $\gamma$ is the adiabatic index of the gas. The quantity $P_{1}$ is primarily due to the dynamic pressure* of the wind. This is a straightforward extension of the approximation due to Kompaneets (1960). We adopt spherical coordinates $(r, \theta, \phi)$, but suppress the dependence on $\phi$ by assuming that $\rho_{0}$ is cylindrically symmetric (this assumption is not necessary for the calculation of the shock propagation, but it appears to do very well for all PNs for which we have enough detailed information). Furthermore, we assume that the RGE gas was deposited by a stationary supersonic wind, so that we can write, to a good approximation,

$$
\rho_{0}=\frac{\rho_{*}}{A(\theta)}\left(\frac{r_{0}}{r}\right)^{2} \text {. }
$$

The function $r(\theta)$ describing the shape of the shock front is a solution of the equation

$$
\frac{\partial r}{\partial t}=\sqrt{K+K\left(\frac{1}{r} \frac{\partial r}{\partial \theta}\right)^{2}}
$$

(I1988). Substitution of Eqs. (1) and (2) gives

$$
\frac{\partial x}{\partial \tau}=\sqrt{A(\theta)\left[+(\partial x / \partial \theta)^{2}\right]},
$$

in which we have used the dimensionless quantities

\footnotetext{
*This implies that, for a constant wind speed and density, $P_{1}$ secularly decreases as the nebula expands. Accordingly, the timescale $\tau$ depends on the volume encompassed by the shock (cf. Kompaneets 1960). We have not taken this into account because we are primarily interested in the morphology here; the time dependence of $P_{1}$ merely changes the time when a certain shock configuration is reached, not the configuration itself. Moreover, it is not at all likely that the density and the velocity of the fast wind are time independent. Technically, $P_{1}(\tau)$ could be admitted by a suitable redefinition of $\tau$, but too little is known about the actual time development of the fast wind to warrant the effort.
}

$$
x \equiv \log \left(r / r_{0}\right) ; \quad \tau \equiv \frac{t}{r_{0}} \sqrt{\frac{\gamma+1}{2}} \frac{P_{1}}{\rho_{*}} .
$$

Due to the inverse-square behavior of the $R G E$ gas density, we have obtained an equation for the propagation of the shock that is particularly easy to solve: Eq. (4) can be decomposed by means of separation of variables. For, if we assume that $x$ is the sum of a function of $\tau$ and a function of $\theta$, we readily find that

$$
x=E \tau-\int \sqrt{E^{2} / A(\theta)-1} d \theta+g(E) .
$$

Here $E$ is an arbitrary constant, and $g(E)$ an arbitrary function thereof. From this so-called "complete integral" we may obtain other solutions of Eq. (4) by taking the envelope of all functions in Eq. (6) for a given range of values of $E$ (Courant and Hilbert 1968, Part II, Chap. I, Sec. IV). Because the stellar wind is presumably spherically symmetric (although this remains to be proven observationally), $x$ must be constant (which we may take to be zero) at $\tau=0$, for all values of $E$; therefore, in our applications we have $g(E)=0$. Note, however, that other initial conditions may be chosen; for example, if we wish to calculate how the shock of an E type PN moves after breaching the outer layers of the RGE, we may pick $g(E)$ to describe the shape of an elliptical shock at $\tau=0$. Once the RGE density distribution has been specified by means of the function $A(\theta)$, Eq. (6) can be evaluated by a simple quadrature for any desired value of $E$. (We have found Simpson's Rule accurate enough for all our applications.)

Because it is utterly unknown how the RGE is deposited (see Sec. V for references to some possible mechanisms), and $a$ fortiori how it acquired its $\mathrm{e} / \mathrm{p}$ ratio, we had to make a guess at the function $A$; a plausible expression is

$$
A(\theta)=1-\alpha+\alpha \exp [\beta \cos (2 \theta)-\beta] \text {. }
$$

Here $\alpha$ and $\beta$ are constants that describe the $\mathrm{e} / \mathrm{p}$ ratio and the steepness of $A(\theta)$. For small values of $\beta$, we have used the asymptotic form of Eq. (7):

$$
A(\theta)=1-\beta+\beta \cos (2 \theta)=1-2 \beta \sin ^{2} \theta .
$$

Accordingly, small values of $\beta$ represent density distributions of which the cross-section contours are ellipses with axial ratio (pole/equator) $=\sqrt{1-2 \beta}: 1$. Some examples of $A(\theta)$ and the density distribution derived from it are given in Figs. 3 and 4. Note that small values of $\alpha$ produce a density that has an $\mathrm{e} / \mathrm{p}$ ratio close to unity. Large values of $\beta$ give a density that is quite independent of $\theta$ near the equator, but that drops steeply in a narrow zone near the pole, thus producing an almost spherical RGE cross section with a "funnel" centered on the symmetry axis.

In Fig. 5 we show the time evolution of shock fronts travelling through the density distributions prescribed by Eqs. (7) and (8), for various values of $\alpha$ and $\beta$. Note that all the shocks evolve towards a fixed shape in a finite time, typically on the order of $\tau=4$. We will encounter this phenomenon again when discussing synthetic PN spectra and isovelocity images. This behavior is generic, and can be shown to be due to the fact that the values of $E$ in Eq. (6) always span a finite range for a bounded function $A(\theta)$ (I1988, Sec. 3.2). We will see later that Eq. ( 7 ) with $\beta \approx 4$ or more (see Figs. 3 and 4 ) gives a good fit to the observed nebular shapes: the RGE that surround butterfiy PNs are not spheroidal, but rather resemble spheres with an axial funnel. 


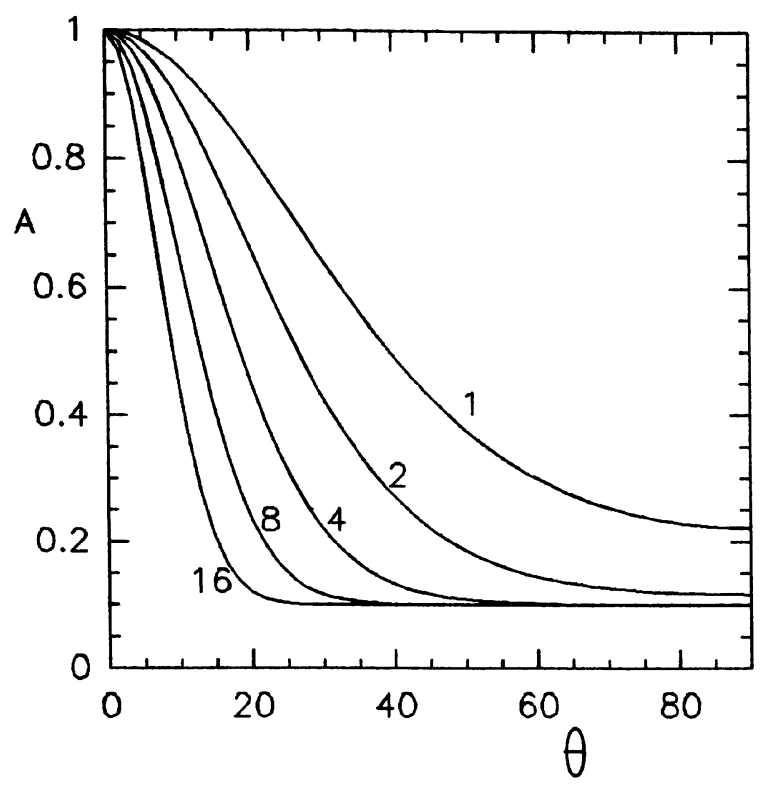

FIG. 3. Graphs of the function $A(\theta)$ from Eq. (7), for $\alpha=0.9$. Values of $\beta$ are indicated on the curves. The RGE density is inversely proportional to $A$.

\section{b) Line Shapes}

The solution in Eq. (6) of the shock-propagation equation allows us to find the shock velocity from the expressions

$$
\begin{aligned}
& D_{\mathrm{R}}=\sqrt{A(\theta)} \sin (\theta+\psi), \\
& D_{z}=\sqrt{A(\theta)} \cos (\theta+\psi), \\
& \psi=-\arctan (\partial x / \partial \theta)
\end{aligned}
$$

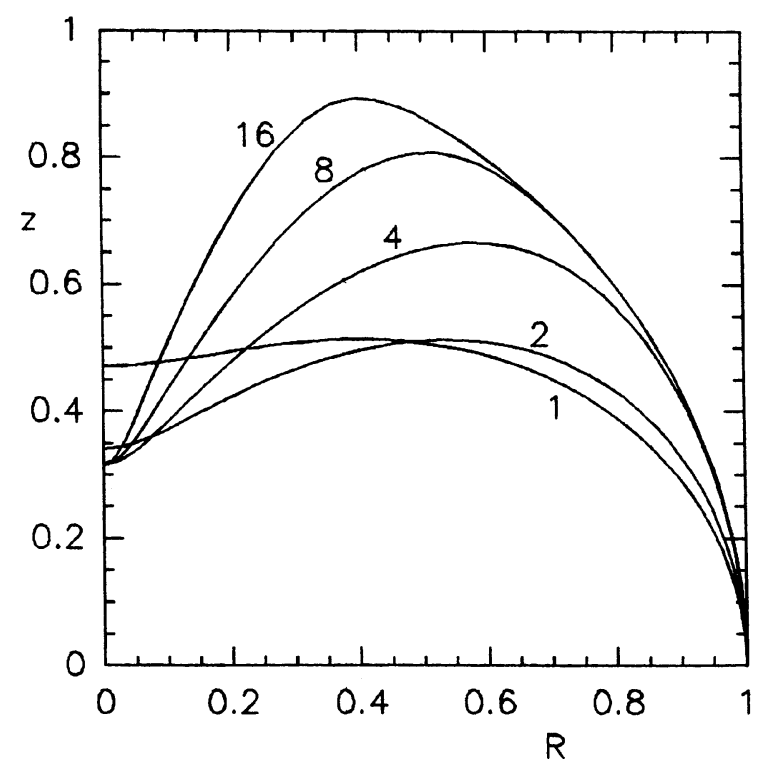

FIG. 4. Cross sections of the RGE density distribution as derived from the function $A(\theta)$. Because the density is self-similar, all equidensity contours have the same shape (although on different linear scales). The $z$ axis is the axis of cylindrical symmetry; the $R$ axis lies in the equatorial plane. Values of $\beta$ are indicated on the curves.
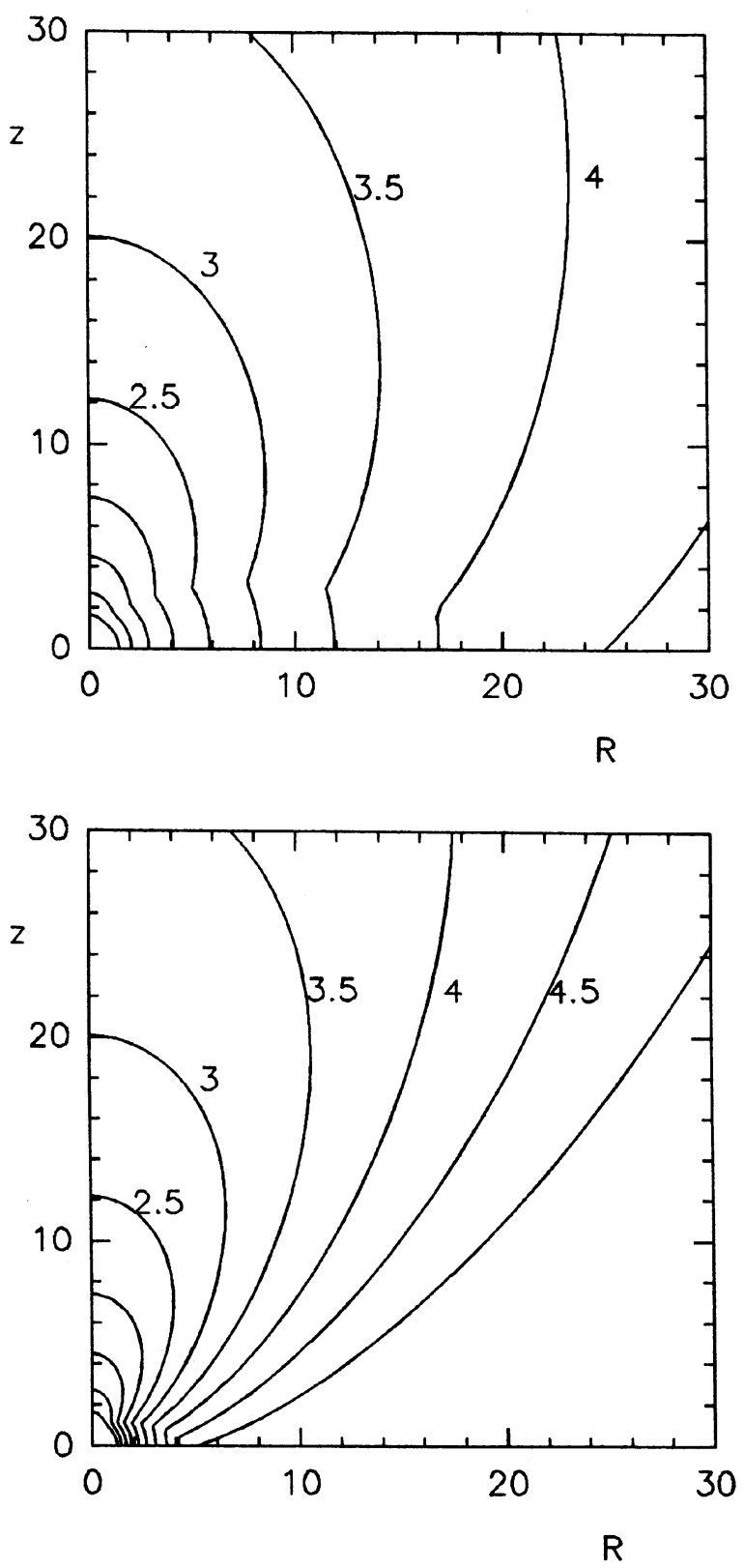

Fig. 5. Evolution of the shock solutions in Eq. (6), with RGE density contours prescribed by Eq. (7), for $\beta=4$. The top frame shows the case $\alpha=0.5$, the bottom frame has $\alpha=0.9$. Dimensionless times $\tau$ are indicated on the curves. Notice the development of the equatorial cusp; once this cusp has formed, the shape of the shock no longer changes (although it increases secularly in linear scale; see the text).

(I1988, Sec. 3.2). Here $D_{\mathrm{R}}$ is the component of the velocity parallel to the equatorial plane of the RGE, and $D_{z}$ is the component along the symmetry axis. In two-dimensional spectroscopy, this velocity is usually the primary observable (see Sec. II above), so we must investigate in detail how the shocks described by Eq. (6) present themselves in kinematic PN images. To this end, we have calculated the positionvelocity images of a series of PN shocks, in an effort to discover the generic shapes of such images, to find features that 
are good diagnostics of aspherical shock propagation, and to compare all these with the observations. The images are stored in the form of a "data cube," a three-dimensional figure with two spatial axes and one velocity axis. Every point within the cube has an associated scalar that indicates the intensity of the radiation received at its particular position and velocity.

When a thin slice, centered on a plane that is parallel to the velocity axis, is cut out of this cube, one obtains an image that represents a long-slit spectrum of the nebula; the width and the orientation of the slit are given by the intersection of the slice with a plane perpendicular to the velocity axis. When a similar slice is cut out of the cube perpendicular to the velocity axis, one obtains an isovelocity image of the nebula, with a center and width that are determined by the position of the slice along the velocity axis (cf. BPI1987 and Sec. IV $c$ below). All slices in our theoretical plots have a width that equals twice their spacing (Nyquist sampling).

We filled our synthetic data cubes as follows. For a given function $A(\theta)$, we evaluated Eq. (6) over the full range of $E$ allowed by the condition that the shock be initially spherical. Then we calculated the shape of the shock for a range of values of the dimensionless time $\tau$, by forming the envelope of all $x(\tau, \theta, E)$ (cf. I1988, Sec. 2.3). From Eq. (9) we determined the velocity vectors along the shock front. The maximum value of the radius of the shock was taken as the unit of length, while the maximum speed became the unit of velocity. Assuming a value of the tilt angle of the RGE symmetry axis with respect to the line of sight, we calculated the radial velocities along the front as projected in the sky, thus obtaining a surface in the space spanned by the plane of the sky and the radial velocity. This surface was then enclosed by a cube centered on the star, with sides of length 2 , completing the construction of the data cube.

A sample of typical synthetic PN spectra is shown in Fig. 6. These are the kind of spectra with which an observer would be confronted before constructing a kinematic data cube. Note, however, that the slit positions in our figures are all parallel to the symmetry axis of the nebula; this orientation is best for showing the peculiar features of the aspherical shock motion. It is obvious from these simulations that most spectral features taken with the slit parallel to the major axis of the RGE, and across the central star, have a pronounced hourglass shape.

For a given RGE distribution, the "waistline" of a spectral line becomes more narrow at later times, and is also more narrow for $\mathrm{RGE}$ densities that have a larger $\mathrm{e} / \mathrm{p}$ ratio. Indeed, because every shock in our models evolves toward a fixed shape, determined only by the function $A(\theta)$ which describes the RGE density distribution, the relative width of the waistline is a good indicator of the equator-to-pole contrast, provided that the $\mathrm{PN}$ is old enough to have approached its asymptotic shape. This approach is quite clear in Fig. 5 (see also Figs. 7 and 8, below).

When the slit is placed more towards the edge of the nebula, or when the $\mathrm{e} / \mathrm{p}$ ratio is not too large, the lobes of the hourglass become narrower. However, their presence is still seen in the line shape, albeit in a devious way: due to the combination of the shock motion and the inclination of the nebular axis, the spectral lines appear to be tilted, giving a false impression of rotation, especially in spectra with moderate resolution. Such spectral lines look like boxlike, hollow, tilted ellipses, usually with ends that are rather fainter than the sides. This is the phenomenon called "false rotation" in our discussion of the observations in Sec. II.

\section{c) Isovelocity Images}

An isovelocity image is a slice through the kinematic data cube perpendicular to the velocity axis. It represents an image of the PN as it would be seen in the sky by a detector tuned to an extremely narrow spectral band. Synthetic isovelocity images of elliptical PNs were presented by BPI1987, who compared these images with observations of $\mathrm{E}$ type PNs. They found that the kinematic model in which a fast spherical wind interacts with a cylindrically symmetric RGE accounts adequately for the observational data, provided that the presence of the "soft bullets" was taken into account by adding the bow shocks they caused to the model velocity field.

A sample of typical synthetic isovelocity images of butterfly PNs is shown in Fig. 7. The influence of the RGE density function $A(\theta)$ in Eq. (7) can be distilled from Fig. 8, which shows the evolution of the nebular shape as a function of time and of the parameter $\beta$. Some of the characteristic features of these images are reminiscent of those found by BPI1987 in elliptical PNs, namely a central expanding sphere centered on the star, with redshifted and blueshifted patches further along the symmetry axis. In our butterflies, however, the presence of the ansae that often dominate the appearance of E type PNs is restricted to a small fraction of parameter space. The low-ionization clouds at the tips of these ansae are absent in butterflies.

Thus, the off-center patches in butterfly PNs can be entirely ascribed to expanding bubbles above and below the equatorial density enhancement in the RGE. The extent of the patches in velocity space, compared with the expansion velocity at the equator, can be traced directly to the $\mathrm{e} / \mathrm{p}$ ratio: in RGEs where the equatorial belt is very dense, a sphere with missing polar caps expands slowly in the equatorial belt, while above and below it, attached to the place where the polar caps ought to be, the bubbles expand quickly. Moderately dense equatorial belts produce patches in the isovelocity images that span a velocity range more nearly equal to the expansion of the sphere.

\section{d) Very Young, Very Old, or Very Tilted Butterflies}

In the above, we have presented synthetic data cubes for butterflies that mostly show a well-developed bipolar appearance. The reason for this is that this is what they generally look like in actual observations, but there is a selection effect: unless the bipolarity is quite marked, such nebulae would not be classified as butterflies. Figure 7 shows that even at early times, when the PNs look almost spherical as seen in the sky, their kinematics (not the morphology) show the influence of the $e / p$ ratio of their RGE density. NGC 1535 is a good example of a round, compact PN which has a conspicuous and "unexpected" kinematic symmetry axis (Clayton 1988).

Even in such early stages, the hydrodynamics of the propagating shocks reveals a very visible effect of density gradients. Planetary nebulae with strong RGE asphericity should have spectra that look much more hourglass shaped than those of E types, which would appear rather elliptical, even though all young PNs look very much alike morphologically (namely, elliptical or, at most, mildly peanut shaped). Thus, young butterfly PNs might be selectively underrepresented in observational samples of kinematic data.

In general, equatorial cusps (such as those seen in NGC 650-1) indicate that the PN is quite old, because it has closely approached its asymptotic shape; conversely, a rounded 

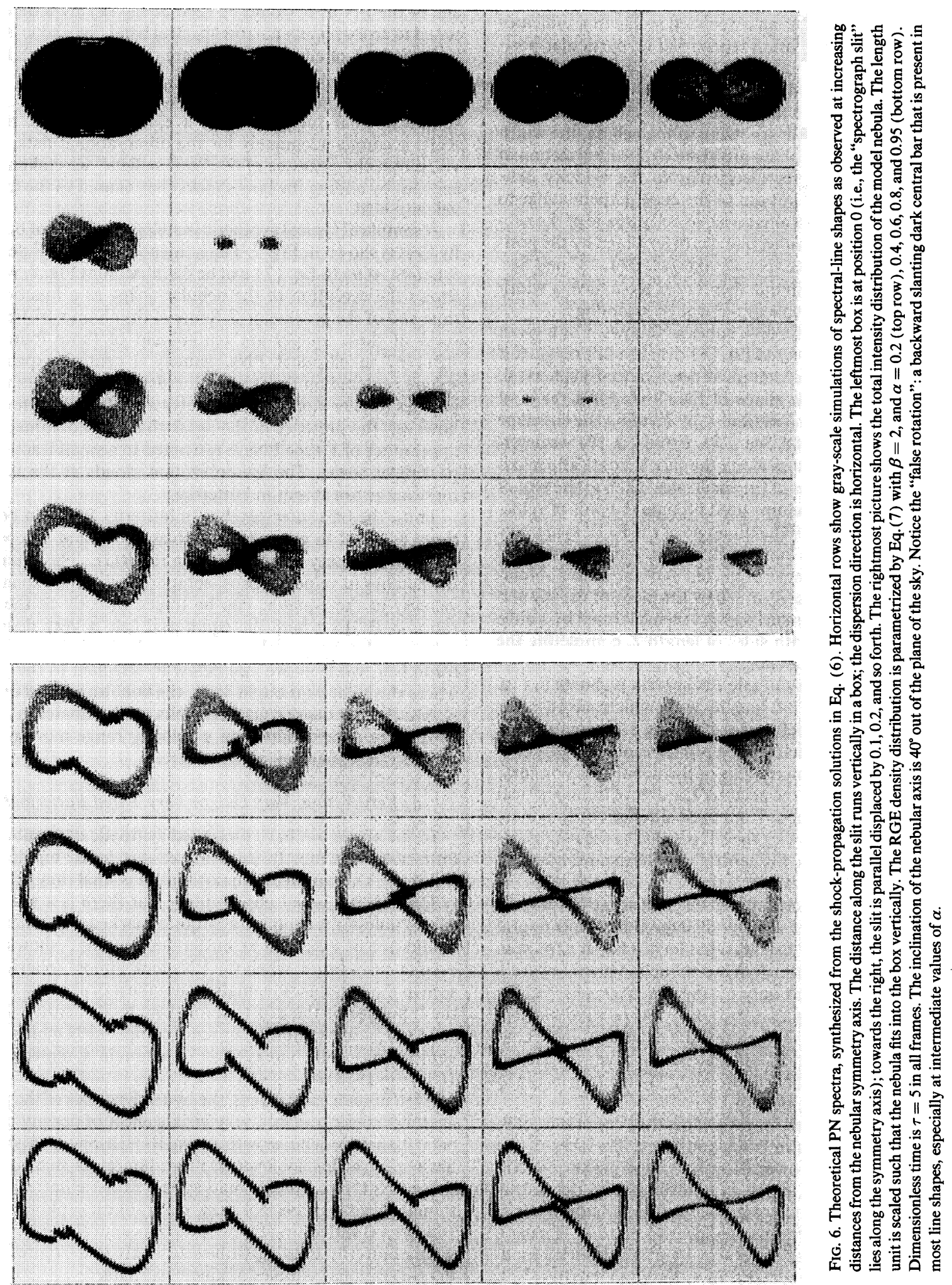


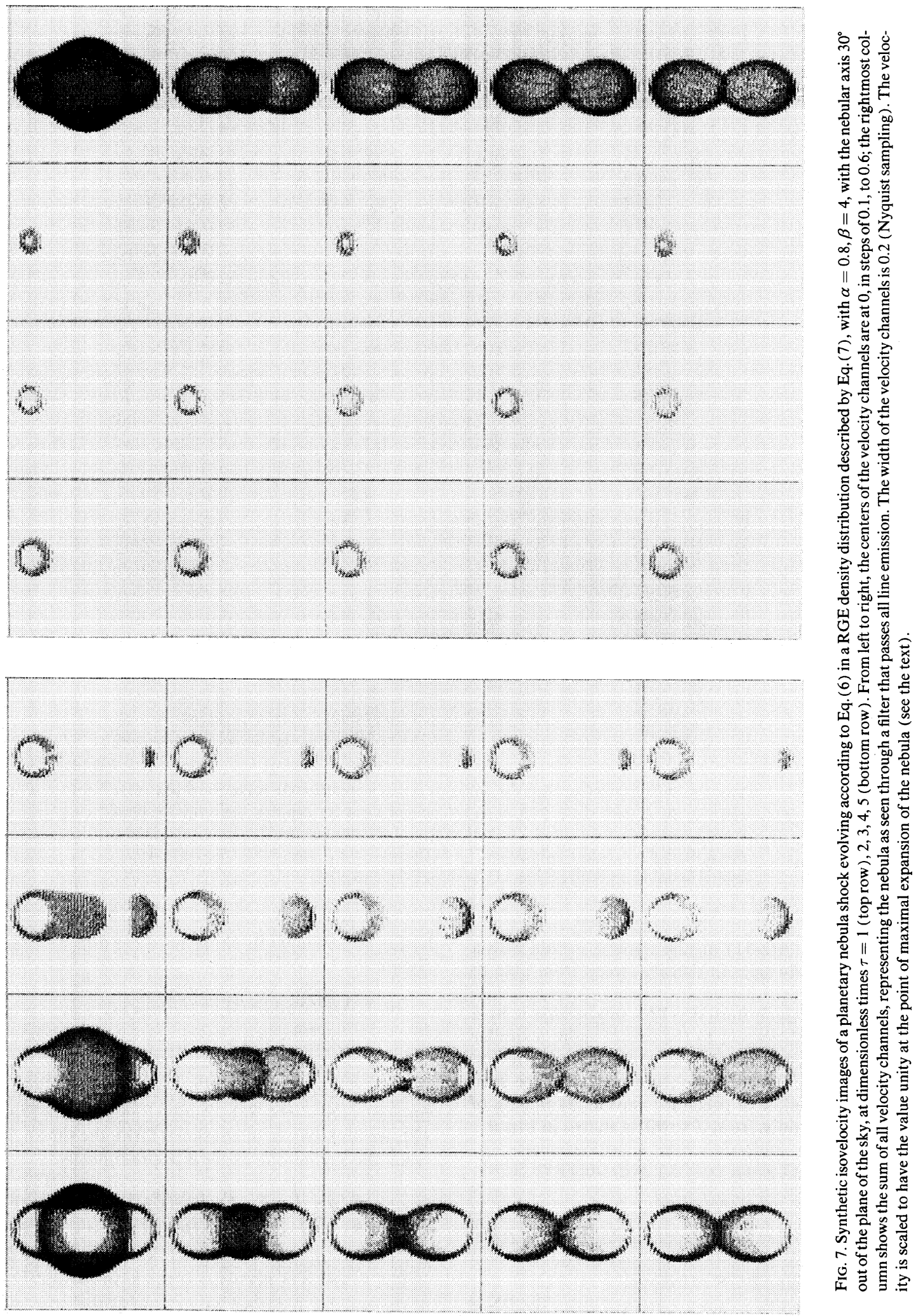




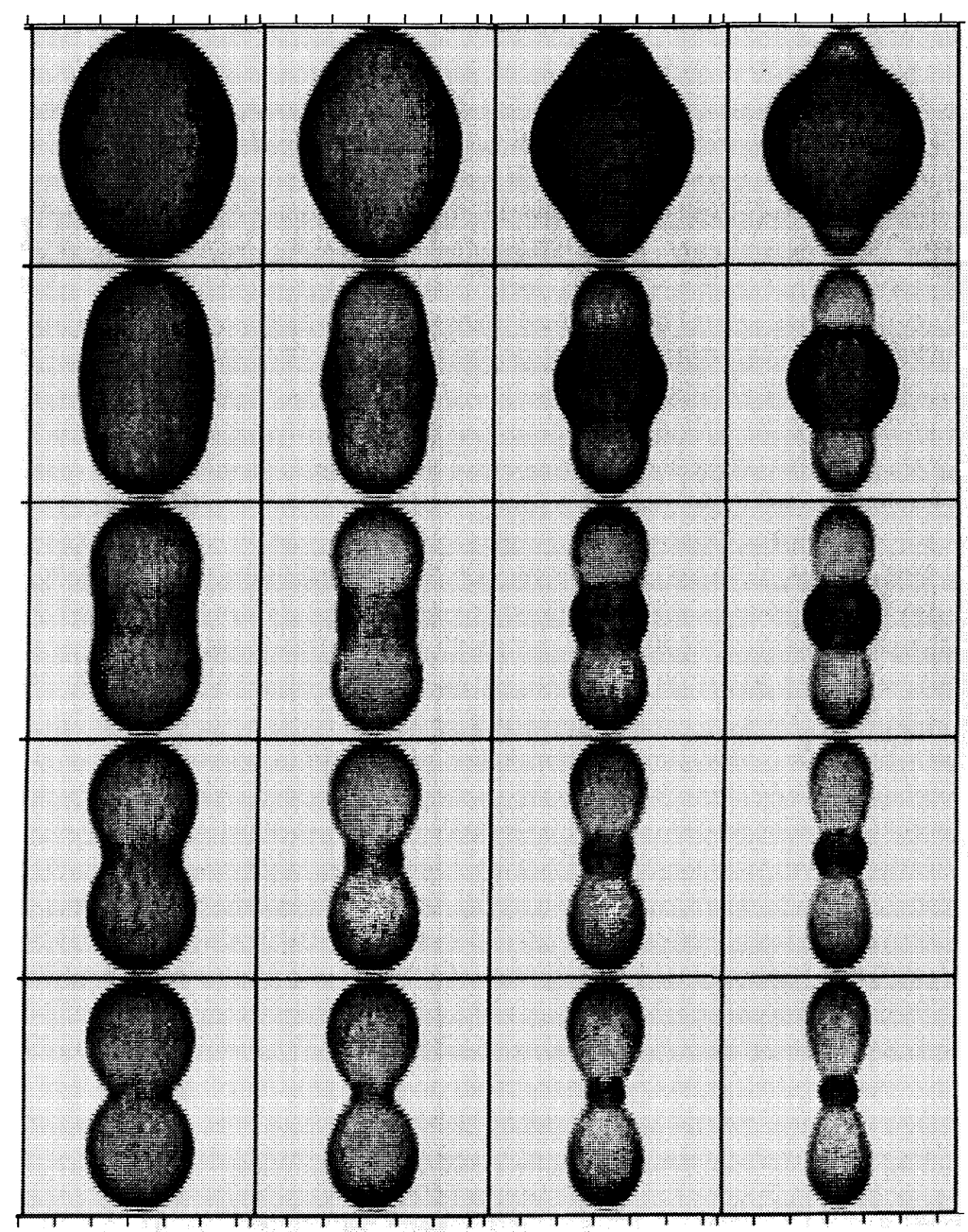

FIG. 8. Evolution of nebular shapes as a function of time and of the RGE parameter $\beta$ (Eqs. (6) and (7)). In all frames, $\alpha=0.95$, or an e/p ratio of about 20 . Top row, dimensionless time $\tau=0.5$; next rows, $\tau=1,1.5,2,2.5$ (bottom row). Left-hand column, $\beta=1$; second column, $\beta=2$; third, $\beta=4$; right-hand column, $\beta=8$. The parameter $\beta$ describes the steepness of the central funnel of the RGE (cf. Fig. (11)). The nebular axes are $30^{\circ}$ out of the plane of the sky.

waistline (as seen in M2-51) means a young nebula with a RGE that has a fairly smooth density sweep from pole to equator.

Another way in which butterfly PNs might hide is by a large inclination with respect to the line of sight; a similar case was discussed by BPI1987, who confirmed that the Eskimo Nebula, NGC 2392, is an intermediate E type seen nearly end-on. A sample of predicted isovelocity images of sutterflies at inclinations from $0^{\circ}$ to $90^{\circ}$ is shown in Fig. 9.

\section{COMPARISON BETWEEN THEORY AND OBSERVATIONS}

\section{a) Morphology}

Butterfly PNs that are seen perpendicular to the symmetry axis of their RGE reveal their age and the density contrast in the surrounding RGE as follows.

If the equatorial outline shows a marked cusp, the PN is old, in the sense of having (nearly) reached its asymptotic shape. As was shown by I1988, an initially spherical winddriven shock in a RGE with a density distribution described by Eq. (2) changes its shape until the dimensionless time $\tau$ equals

$$
\begin{aligned}
\tau_{\text {crit }}= & (\sqrt{A(0)}-\sqrt{A(\pi / 2)})^{-1} \\
& \times \int_{0}^{\pi / 2} \sqrt{A(0) / A(\theta)-1} d \theta .
\end{aligned}
$$

For purposes of estimation, let us assume that $A(\theta)$ is a step function at $\theta=\pi / 4$. Substituting $\tau$ and $A$ from Eqs. (5) and (2), we find that, in dimensional form, the time $t_{\mathrm{c}}$ needed to reach the asymptotic shape is approximately

$$
t_{\mathrm{c}}=\frac{\pi}{4} R \sqrt{\frac{2}{\gamma+1} \frac{\rho_{\mathrm{e}}}{P_{1}}} \frac{\sqrt{\rho_{\mathrm{e}} / \rho_{\mathrm{p}}-1}}{\sqrt{\rho_{\mathrm{e}} / \rho_{\mathrm{p}}}-1},
$$

where the subscript e refers to the value on the equator at radius $R$, and $\mathrm{p}$ to the value at the pole. Because the density at the equator is rather larger than at the pole, at least for butterfly PNs, we may approximate

$$
t_{\mathrm{c}} \approx R \sqrt{\rho_{\mathrm{e}} / P_{1}} \text {. }
$$




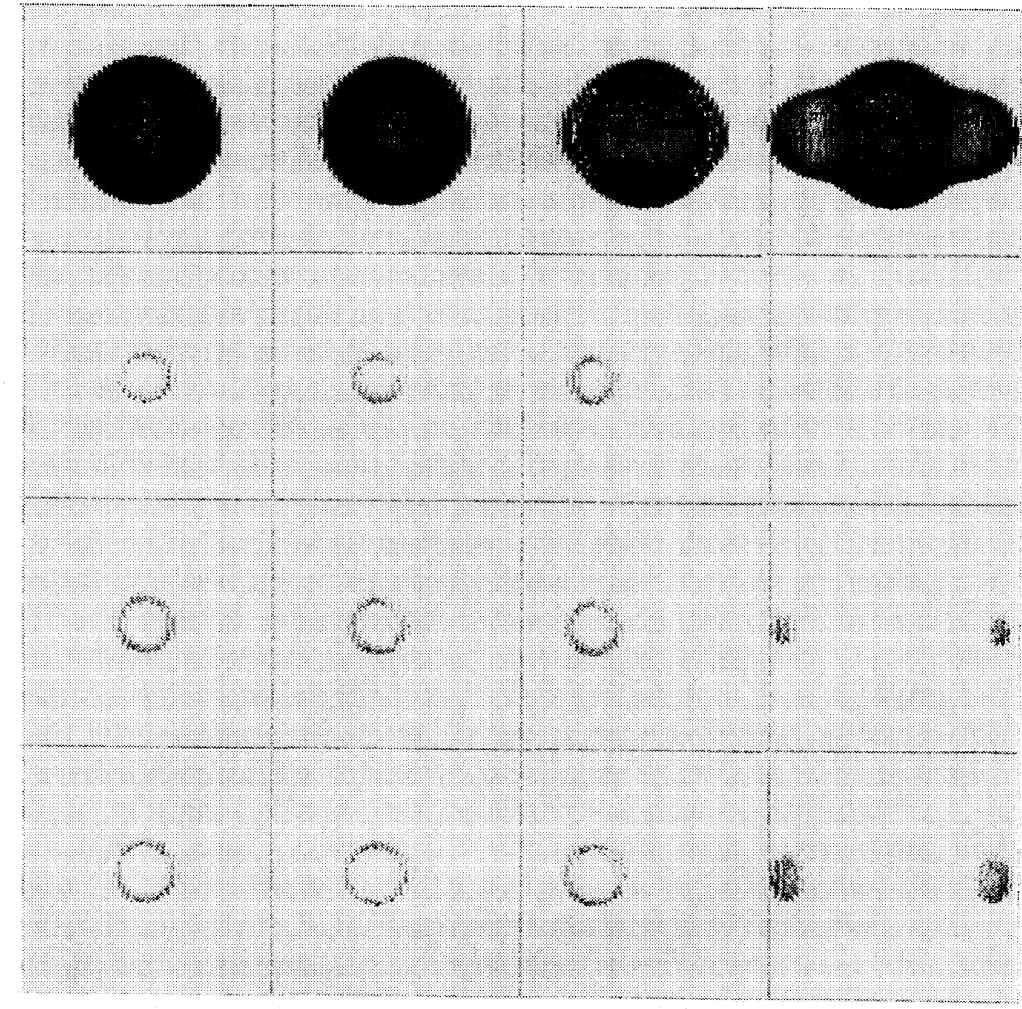

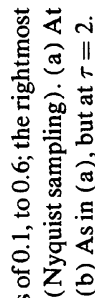

总

कि

월

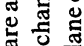

능

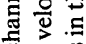

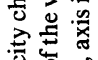

过

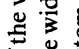

空

路

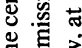

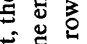

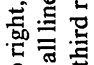

空

을

홍

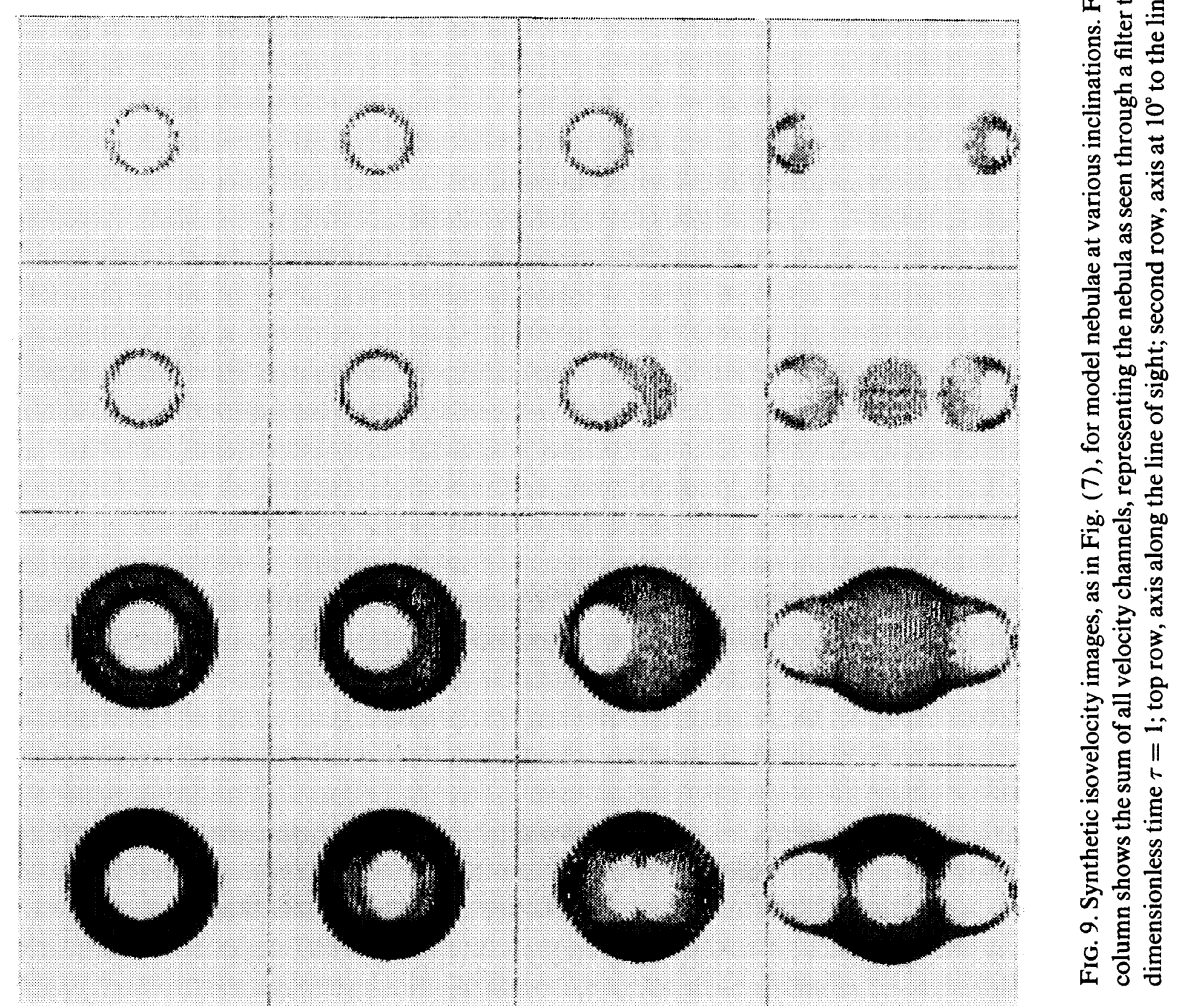



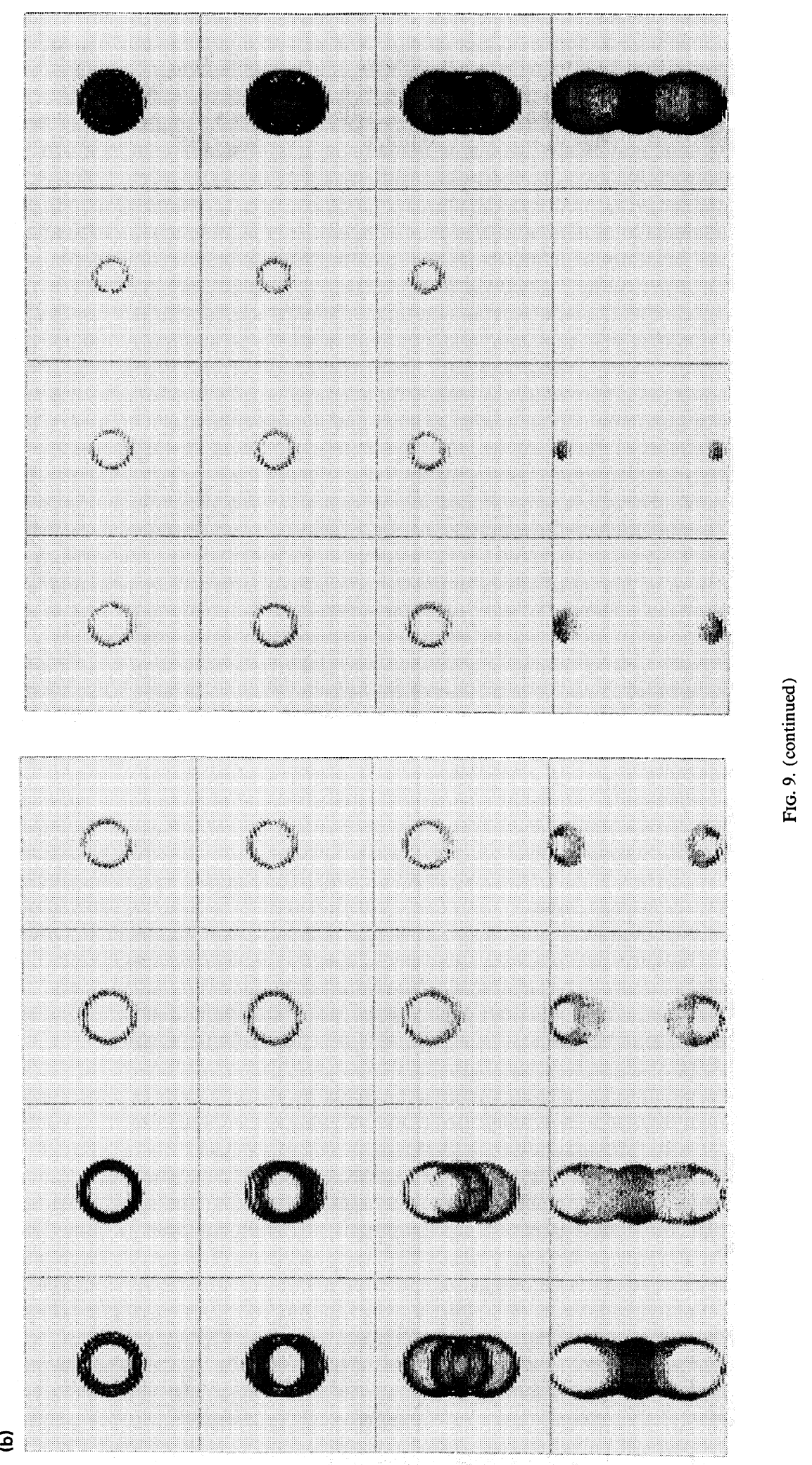
Substituting for $P_{1}$ the dynamic pressure $\rho_{\mathrm{w}} v_{\mathrm{w}}^{2}$ of the stellar wind, we finally get

$$
t_{\mathrm{c}} \approx \frac{R}{v_{\mathrm{w}}} \sqrt{\rho_{\mathrm{e}} / \rho_{\mathrm{w}}} \text {. }
$$

Upon inserting plausible values for the wind speed $v_{\mathrm{w}}$ $=2000 \mathrm{~km} \mathrm{~s}^{-1}$, the inner radius of the cusp $R=0.2 \mathrm{pc}$, and the ratio of the density of the RGE and the stellar wind at the equator $\rho_{\mathrm{e}} / \rho_{\mathrm{w}}=2500$, we find that roughly $t_{\mathrm{c}}=5000 \mathrm{yr}$.

Consequently, any butterfly PN that shows a sharp equatorial cusp is expected to be on the order of 5000-10 $000 \mathrm{yr}$ old. This should be testable by observing the position of the central star in the Hertzsprung-Russell diagram, because a young white dwarf may evolve noticeably on this timescale.

On the other hand, if the $\mathrm{e} / \mathrm{p}$ ratio $\rho_{\mathrm{e}} / \rho_{\mathrm{p}}$ is only slightly larger than unity, as postulated for the RGEs of elliptical PNs, Taylor expansion of Eq. (11) shows that the time needed to establish the asymptotic shape of the shock scales according to

$$
t_{\mathrm{c}} \propto \sqrt{1-\rho_{\mathrm{e}} / \rho_{\mathrm{p}}} .
$$

Therefore, planetary nebulae that form in RGEs with an $\mathrm{e} / \mathrm{p}$ ratio close to unity will not develop equatorial cusps: their outer shocks will remain elliptical, or at most become mildly peanut shaped, before the shock reaches the outer parts of the RGE (e.g., NGC 40).

Can we expect butterfly PNs to reach their asymptotic shape before the shock breaches the outermost radius of the RGE? At an average speed of $50 \mathrm{~km} \mathrm{~s}^{-1}$, the shock travels about $0.25-0.5 \mathrm{pc}$ along the symmetry axis during the time needed to establish the asymptotic shape. Because typical RGE radii are larger than this, the majority of butterfly PNs that have breached the confines of their RGE will show equatorial cusps (indicating that the asymptotic shape has been reached-NGC 2346 is a good example of this type of situation).

The overall shape of the butterfly shock gives information about the RGE density distribution. For example, if we compare the shock cross sections at a fixed time (say $\tau=3$ ) in Fig. 7 , we see that densities with a larger value of $\beta$ (that is to say, those with a smaller "angular scale length": a steeper drop of $A(\theta)$; see Figs. 3 and 4) produce more elongated shock shapes. Increasing the value of $\alpha$ (producing a larger $\mathrm{e} / \mathrm{p}$ ratio) dramatically increases the equatorial constriction, as a comparison of Figs. 6 and 7 shows, but it turns out that the cross section of the bubble that rises above the equatorial belt is not much influenced.

This is not entirely unexpected: if the region above the equator were as good as empty, we should simply get a pair of expanding spherical shocks above and below the equatorial belt, with their centers on the symmetry axis but away from the central star. Quantitatively, this can be seen as follows. The cylindrical radius $R$ of the bubble cross section obeys

$$
R \equiv r(\theta) \sin \theta
$$

where $r(\theta)$ follows from Eq. (6). Taking the derivative of $R$ with respect to $\theta$, we find that $R$ is maximal when

$$
\partial \log r / \partial \theta=-\operatorname{cotan} \theta \text {. }
$$

Using Eqs. (2) and (6), we conclude that the cross section of the asymptotic shock with a plane parallel to the equator of the RGE is largest when $\theta$ is a solution of

$$
\operatorname{cotan} \theta=\sqrt{\rho(\theta) / \rho_{\mathrm{p}}-1} \text {. }
$$

Here $\rho_{\mathrm{p}}$ is the RGE density at the pole. If $\theta_{\mathrm{m}}$ is the solution of Eq. (16), the corresponding cross section $R_{\mathrm{m}}$ of the bubble, divided by the radius $r_{\mathrm{p}}$ of the bubble along the polar axis, is

$$
R_{\mathrm{m}} / r_{\mathrm{p}}=\sin \theta_{\mathrm{m}} \exp -\int_{0}^{\theta_{\mathrm{m}}} \sqrt{\frac{\rho(\theta)}{\rho_{\mathrm{p}}}} d \theta .
$$

This quantity is an observable: in a given butterfly $\mathrm{PN}$, we can measure the maximum distance $2 r_{p}$ (along the symmetry axis and through the central star) between the farthest points of the shock, and the maximum width $2 R_{\mathrm{m}}$ of the bubble perpendicular to the axis. As can be seen from Eq. (18), the RGE density distribution $\rho(\theta)$ occurs under a square root and in an integrand, so that $R_{\mathrm{m}}$ depends only weakly on the peculiarities of the RGE density. Accordingly, butterfies are expected to be fairly plump figure-eight shapes; an appreciable elongation (i.e., a small value of $R_{\mathrm{m}} / r_{\mathrm{p}}$ ) requires an extremely large $\mathrm{e} / \mathrm{p}$ ratio. In the examples shown above, this elongation is never less than about 0.3 , even for RGE densities that decrease by a factor of 10 from pole to equator.

\section{b) Synthetic Spectra}

The typical shape of the spectral lines of a butterfly PN seen nearly perpendicular to the RGE symmetry axis is an hourglass with rather straight sides (Fig. 6). The narrowness of the waist of the figure is related to the age of the shock and to the $\mathrm{e} / \mathrm{p}$ ratio of its RGE, in the sense that young PNs (ages less than $0.2 \tau_{\mathrm{c}}$ ) tend to have relatively wide waists, as do PNs that have only a mild RGE gradient (equator: pole $=2: 1$ or so).

Many of our PNs have spectral lines that fit this pattern very well. Especially good examples are:

NGC 2440, H $\alpha$ (Fig. 10 [Plate 35] ), notably at positions $8 " \mathrm{~S}$ through 8 " $\mathrm{N}$; these line shapes are virtually identical with those obtained with Eq. (7) in the case $\alpha=0.9$, $\beta=4.0$, at $\tau$ about 1.5 or 2 , with an axis that is inclined $30^{\circ}-$ $35^{\circ}$ with respect to the plane of the sky.

NGC 6302, [N II] line, slit on the star at P.A. $170^{\circ}$. Although the spectrum is quite blobby, the hourglass outline is unmistakable.

M2-9, $\mathrm{H} \alpha$ and [ $\mathrm{N} \mathrm{II}$ ], all slit positions at P.A. $90^{\circ}$. Because of the considerable elongation of the bipolar shape (length:width $=5: 1$ ), the e/p ratio should be on the order of 15. The parameters needed in Eq. (7) are $\alpha=0.9, \beta=16$, at $\tau=1.5$ and inclination $20^{\circ}$, but these values are uncertain by about $40 \%$.

Examples of spectral lines that show the false rotation phenomenon, i.e., an apparent slant of the spectral line due to a combination of nonuniform bubble expansion and inclination of the RGE axis, are seen in: IC 1747, $\mathrm{H} \alpha$, slit on the star at P.A. $78^{\circ}$; NGC 6781, [N II], slit on the star at P.A. $80^{\circ}$; NGC $6905, \mathrm{H} \alpha$ and [N II], slit on the star at P.A. $80^{\circ}$; NGC 2346, [N II] (Fig. 11 [Plate 36]), slit at 16" E, 8" E, on the star, 8 " W, and $16^{\prime \prime} \mathrm{W}$. The nebula shows rather sharp equatorial cusps and open ends, and should therefore be fairly old; on the other hand, the elongation is not extreme (length:width $=2: 1$ ). Also, there is no indication that the lines have hourglass lobes. This implies that NGC 2346 has a fairly modest $\mathrm{e} / \mathrm{p}$ ratio, on the order of 2.5 .

We would also include NGC 7009, [N II], slit at 3" S, on the star, and 3 " $\mathrm{N}$, in the "false rotation" list. This nebula was included in our previous sample (containing $\mathrm{E}$ type 
PNs), but BPI1987 concluded that their kinematic model did not fit this object exactly: "...the caps extend to larger velocity extremes than do the tips of the ansae [which could be explained by] the ad hoc assumption that the axis of the focused flow wobbles in time." Comparing the line shapes of the inner part of NGC 7009 (away from the ansae) with those of our synthetic spectra, we tend to conclude that the outer shock in this nebula has started to expand faster at its extremities, probably due to incipient breaching of the RGE.

\section{CONCLUDING REMARKS}

We extend the conclusions that BPI1987 reached, for elliptical planetary nebulae, to butterfly PNs. We assert that butterflies can be explained by the same physical mechanism as $\mathrm{E}$ types, namely the propagation of a wind-driven shock through a cylindrically symmetric red giant envelope with a lower gas density at the poles than at the equator. To obtain butterfly PNs instead of $E$ types, the e/p ratio needs to be typically larger than 4 , but otherwise our interpretation is consistent with the existence of a continuum of $e / p$ ratios, ranging from 1 for the (rare) actual spherical PNs, toward 2 for typical ellipticals, to 15 for the most extreme butterflies. Due to the peculiarities summarized in Eq. (18), higher values than these-if they occur-are difficult to assert with certainty.

The comparative ease with which we can reproduce the kinematic images of all these types of nebulae allows us to state with some confidence that $\mathrm{e} / \mathrm{p}$ ratios different from unity are common in RGEs. Moreover, there is direct evidence for disklike structures and/or molecular hydrogen in several PNs (Meaburn et al. 1985; Balick, Gatley, and Zuckerman 1988). Thus, we regard the existence of a continuum of $\mathrm{e} / \mathrm{p}$ ratios as established, even though it is unknown what mechanism of mass loss could be responsible for the deposition of such aspherical RGEs. We eagerly await observations of Miras and OH/IR stars that show evidence for the aspherical outflow that is predicted implicitly by our model calculations.

Our interpretation of PN evolution is consistent with cylindrically symmetric RGEs, which points to a rotation-related mechanism; the precession that appears to be present in some PNs (BP1987) reinforces that view. Several authors have tried to calculate the consequences of stellar rotation for mass loss (e.g., Friend and Abbott 1986; Poe and Friend 1986), and some have considered the outflow from merging binaries (Bodenheimer and Taam 1984; Soker and Livio 1988). None of these mechanisms can be regarded as established, but the $\mathrm{e} / \mathrm{p}$ ratios of actual RGEs are definitely spread over a wide range.

Comparison between our synthetic position-velocity images and actual observations is sometimes difficult because of small-scale inhomogeneities in the nebulae, giving the spectra a "blobby" appearance. Because of this, we have not put much effort into modeling the brightness distribution of the lines or the isovelocity images. More realism could be added by calculating the density and the temperature behind the shock, and the emission measure from these; this is possible without further assumptions, simply by applying the $\mathrm{Hu}-$ goniot jump conditions. Furthermore, absorption through the known RGE density distribution could be taken into account (the clear presence of off-center absorption lines in the stellar spectrum of M2-9 shows that such obscuration can be considerable). However, the attendant problems of radiative transfer in such inhomogeneous density distributions would then have to be solved (methods for this exist: see Icke 1979; Icke, Gatley, and Israel 1980).

An added complication is that not all small-scale inhomogeneities can be ascribed to random fragments. For example, the bright knots in the CCD image of M2-9 are symmetric under reflection with respect to the equatorial plane. This is quite puzzling; it certainly cannot be ascribed to precession, as in the case of NGC 6543 (BP1987), where the ansae are symmetric under point inversion with respect to the central star. Moreover, M2-9 is much brighter on the eastern than on the western side. Possibly, it is embedded in an E-W density gradient.

Having now investigated the applicability of our scheme for aspherical PN evolution to a broad range of morphological types, we need to confirm (by means of numerical hydrodynamics) that the basic features of our interpretation are correct. Trial computations with a cylindrically symmetric flux-corrected-transport scheme indicate that the outer shock evolves as expected, while the inner (reverse) shock indeed exhibits the postulated tendency to become prolate spheroidal. Soker and Livio (1988) have confirmed, by means of numerical models, that focusing along the symmetry axis and subsequent cooling occurs, as postulated by BPI1987.

It is a sincere pleasure to thank the many people at the NOAO who capably assisted with the observations. Financial support for the observations was provided to B.B. and H.L.P. by NSF grant no. AST86-12228 and by the NOAO. Image processing at the University of Washington is made possible by grants from the NSF (AST 83-10552) and the Digital Equipment Corporation. This paper was written while B.B. was a summer visitor at Sterrewacht Leiden, for which financial support was graciously provided by the Netherlands Foundation for the Advancement of Pure Research (N.W.O.) through grant no. B 78-251; by the NSF through grant no. INT 87-14186; and by the North Atlantic Treaty Organization (NATO) through grant no. 0898/87.

\section{REFERENCES}

Acker, A. (1982). In Scientific Aspects of the Hipparcos Space Astrometry Mission, edited by M. A. C. Perryman and T. D. Guyenne, ESA SP-177 (ESA, Noordwijk), p. 199.

Balick, B. (1987). Astron. J: 94, 671 (B1987).

Balick, B. (1988). Astron. J. 97, 476.

Balick, B., and Preston, H. L. (1987). Astron. J. 94, 958 (BP1987).

Balick, B., Preston, H. L., and Icke, V. (1987). Astron. J. 94, 1641 (BPI1987).

Balick, B., Gatley, I. A., and Zuckerman, B. (1988). Preprint.

Bodenheimer, P., and Taam, R. E. (1984). Astrophys. J. 280, 771.

Cerruti-Sola, M., and Perinotto, M. (1985). Astrophys. J. 291, 237.
Clayton, C. A. (1988). Astron. Astrophys. 195, 263.

Costero, R., Tapia, M., Mendez, R. H., Echevarria, J., Roth, M., Quintero, A., and Barral, J. F. (1986). Rev. Mex. Astron. Astrofis. 13, 149.

Courant, R., and Hilbert, D. (1968). Methoden der mathematischen Physik (Springer, Berlin).

Friend, D. B., and Abbott, D. C. (1986). Astrophys. J. 311, 701.

Healy, A. P., and Huggins, P. J. (1988). Astron. J. 95, 866.

Heap, S. R. (1987). Nature 326, 571.

Heathcote, S. R., and Weller, W. G. (1987). In Late Stages of Stellar Evolution, Astrophysics and Space Science Library Vol. 132, edited by S. Kwok and S. R. Pottasch (Reidel, Dordrecht), p. 411. 
Icke, V. (1979). Astron. Astrophys. 78, 352.

Icke, V. (1988). Astron. Astrophys. 202, 177 (I1988).

Icke, V., Gatley, I. A., and Israel, F. P. (1980). Astrophys. J. 236, 808.

Kahn, F. D. (1983). In Planetary Nebulae, IAU Symposium No. 103, edited by D. R. Flower (Reidel, Dordrecht), p. 305.

Kahn, F. D., and West, K. A. (1985). Mon. Not. R. Astron. Soc. 212, 837.

Kohoutek, L., and Surdej, J. (1980). Astron. Astrophys. 85, 161.

Meaburn, J., Walsh, J. R., Morgan, B. L., Hebden, J. C., Vine, H., and Standley, C. (1985). Mon. Not. R. Astron. Soc. 213, 35P.
Perinotto, M. (1983). In Planetary Nebulae, IAU Symposium No. 103, edited by D. R. Flower (Reidel, Dordrecht), p. 323.

Poe, C. H., and Friend, D. B. (1986). Astrophys. J. 311, 317.

Pottasch, S. R. (1984). In Planetary Nebulae, IAU Symposium No. 103, edited by D. R. Flower (Reidel, Dordrecht), p. 175.

Reay, N. K., Walton, N. A., and Atherton, P. D. (1988). Mon. Not. R. Astron. Soc. 232, 615 .

Soker, N., and Livio, M. (1988). Preprint.

Walsh, J. R. (1983). Mon. Not. R. Astron. Soc. 202, 303. 


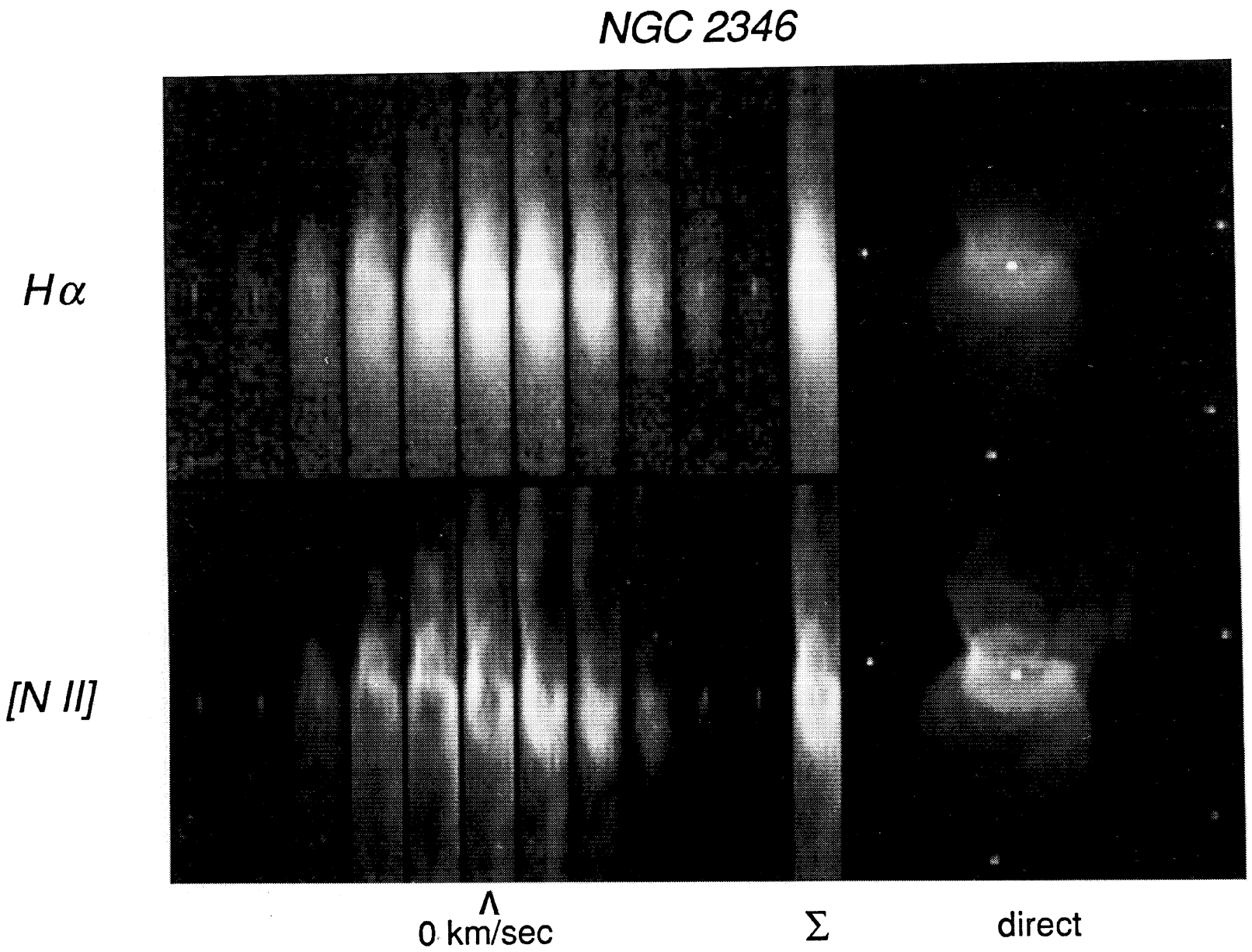

FIG. 1. Kinematic image of NGC 2346 in the lines of $\mathrm{H} \alpha$ (top) and [N II] (bottom). The first 11 frames show the nebula as it would appear through narrowband filters of width and relative offsets of $8.91 \mathrm{~km} \mathrm{~s}^{-1}$. The sixth frame corresponds to zero velocity. North is up in all frames. These frames have been added to produce the twelfth frame, which shows the nebula as it would appear through a filter that passes all line emission. The final frames are undistorted direct CCD images from B1987.

Icke et al. (see page 463) 
(a)

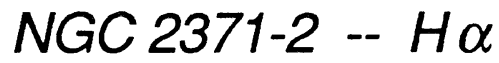
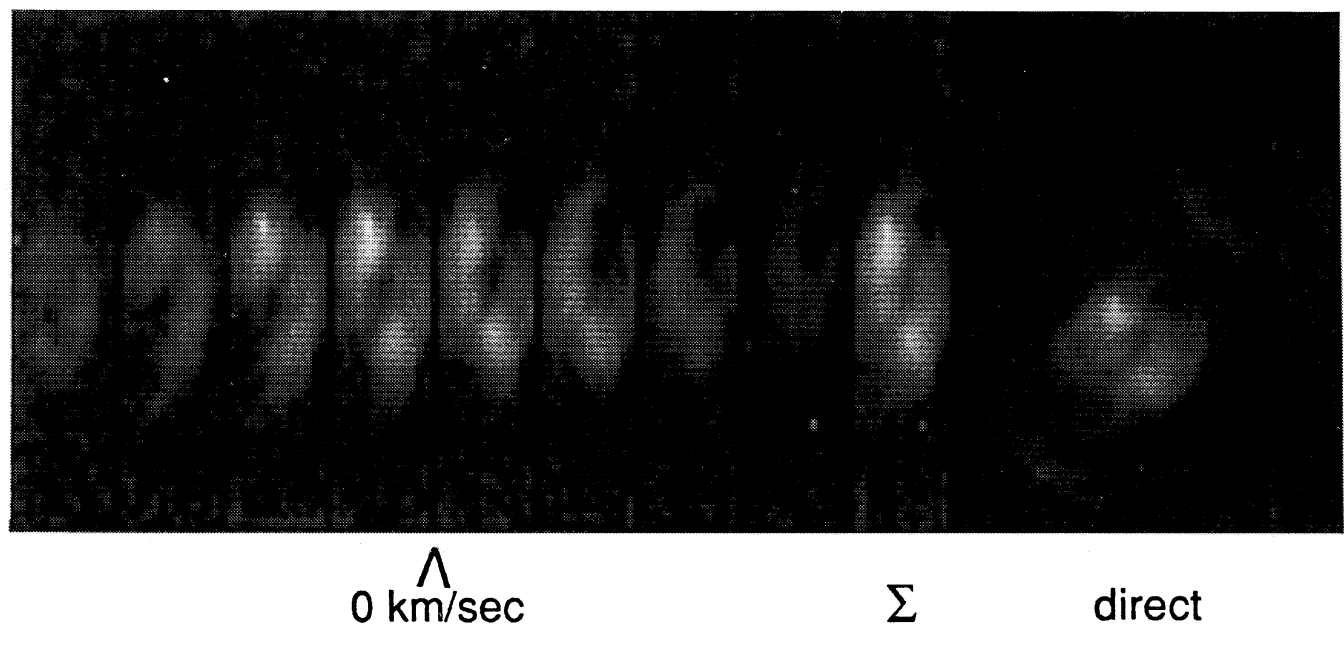

(b)

NGC $2440--[N I I]$

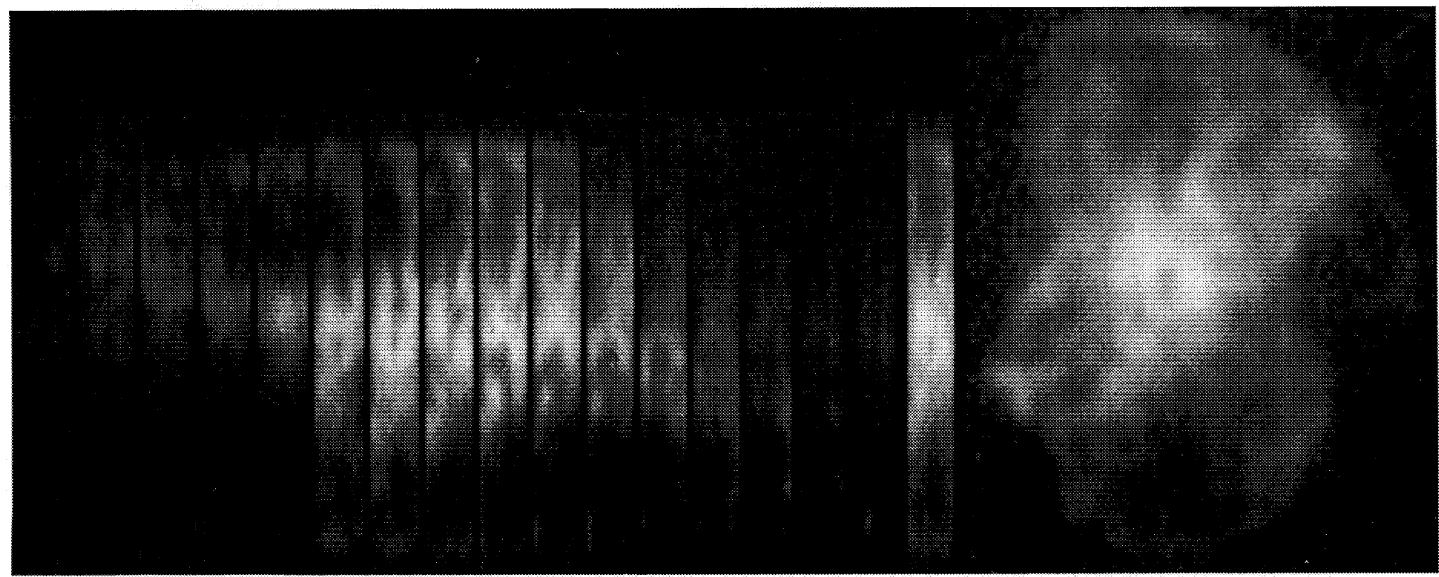

$\Lambda$

$0 \mathrm{~km} / \mathrm{sec}$
$\Sigma$

direct

FIG. 2. (a) Kinematic image of NGC 2371-2 in the line of $\mathrm{H} \alpha$. The first eight frames show the nebula as it would appear through narrowband filters of width and relative offsets of $8.91 \mathrm{~km} \mathrm{~s}^{-1}$. All images are displayed as in Fig. 1. (b) Kinematic image of NGC $2440 \mathrm{in}$ the line of [N II]. The first 16 frames show the nebula as it would appear through narrowband filters of width and relative offsets of 8.91 $\mathrm{km} \mathrm{s}^{-1}$. (c) Kinematic image of NGC 6058 in the line of $\mathrm{H} \alpha$. (d) Kinematic image of NGC 6210 in the lines of $\mathrm{H} \alpha$ and [N II]. (e) Kinematic image of IC 1747 in the line of $\mathrm{H} \alpha$. (f) Kinematic image of IC 5217 in the lines of $\mathrm{H} \alpha$ and [N II] . (g) Kinematic image of J-320 in the line of $\mathrm{H} \alpha$. (h) Kinematic image of M2-9 in the lines of $\mathrm{H} \alpha$ and [N II].

Icke et al. (see page 463) 


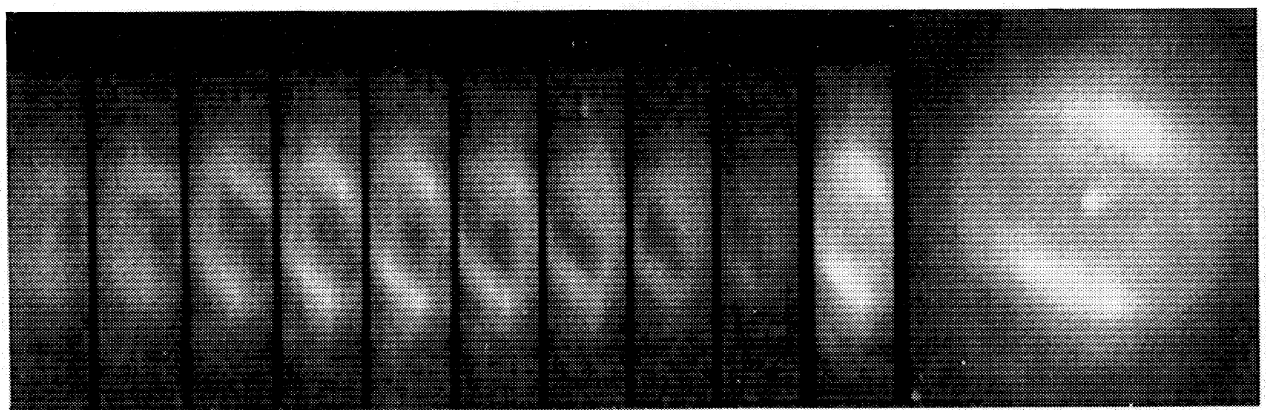

$\wedge$

$0 \mathrm{~km} / \mathrm{sec}$

$\Sigma$

direct

(d)

NGC 6210

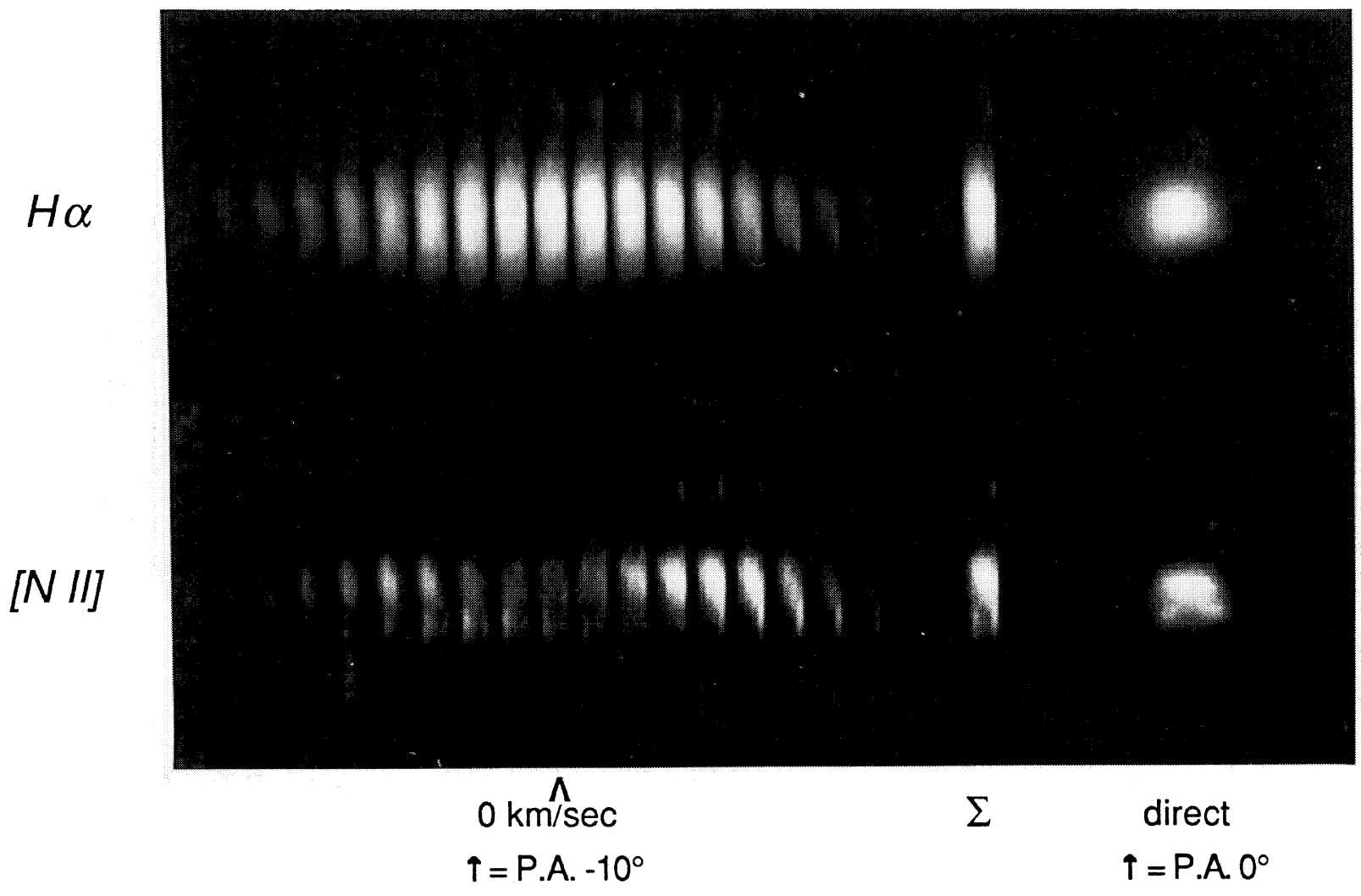

FIG. 2. (continued)

Icke et al. (see page 463) 
(e)

IC 1747-- $H \alpha$

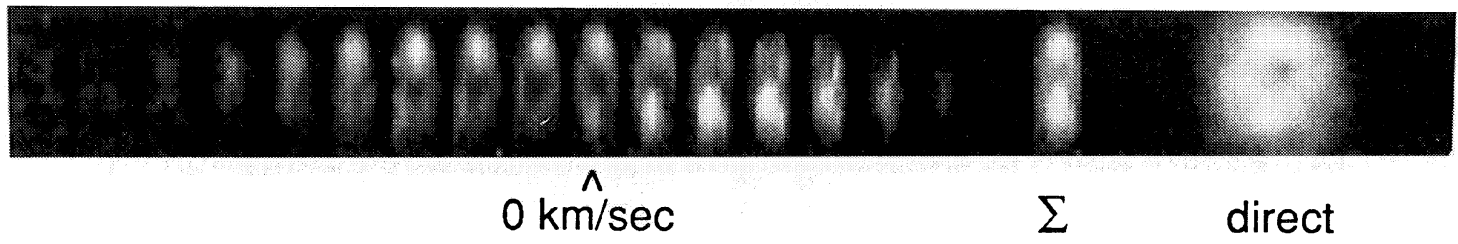

(f)

IC 5217

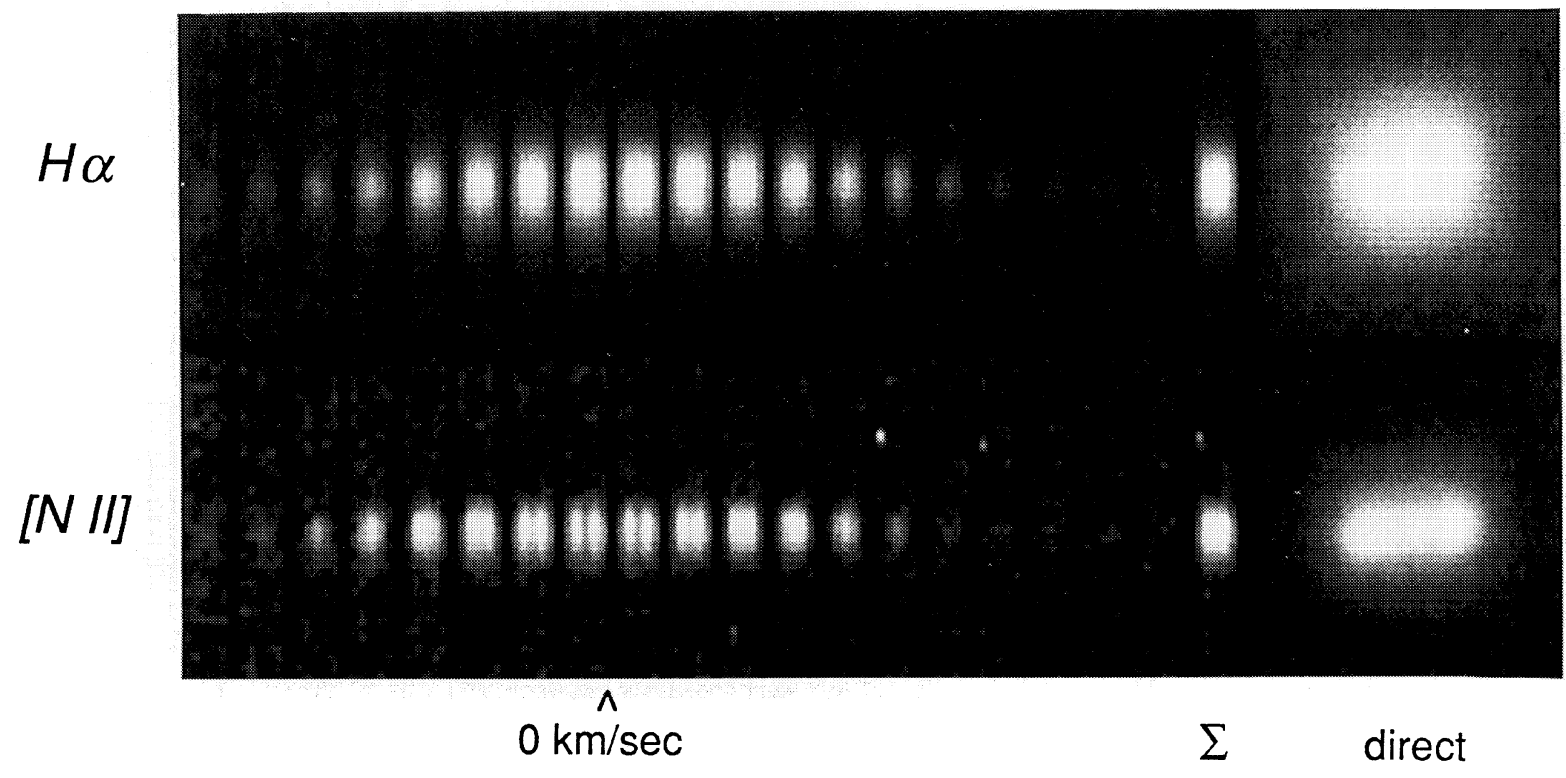

FIG. 2. (continued)

Icke et al. (see page 463) 
(g)

$$
J 320--H \alpha
$$

$$
\begin{array}{r}
\text { velocity }= \\
-80.2 \\
-35.6 \\
\\
+8.9 \\
\\
+53.5 \\
\mathrm{~km} / \mathrm{sec}
\end{array}
$$

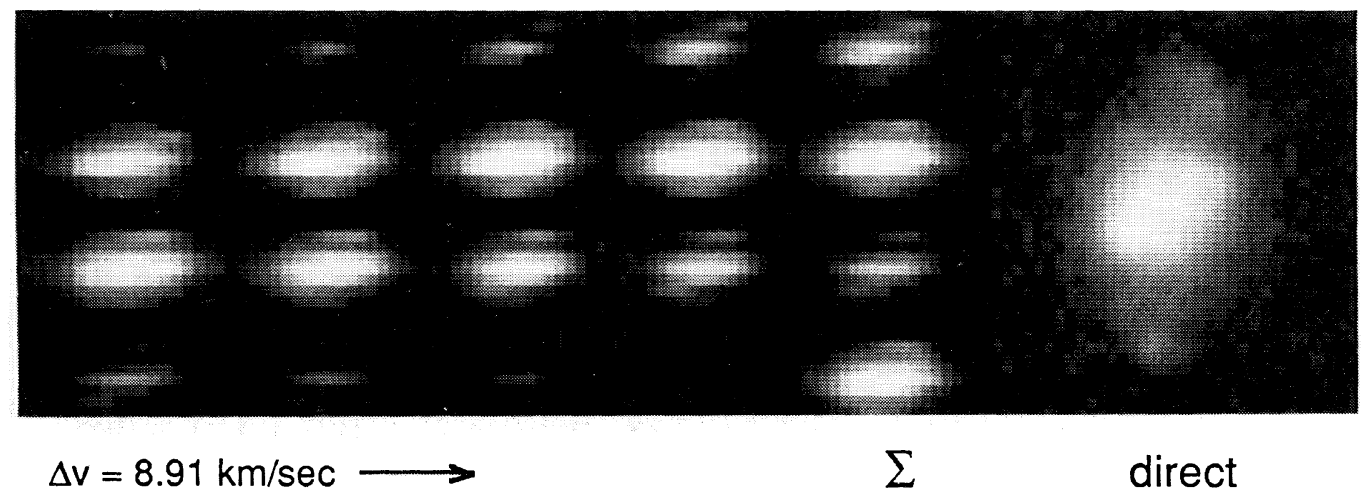

ECHELLE SPECTRA up=north (1"/pixel); right=velocity $(8.91 \mathrm{~km} / \mathrm{sec} / \mathrm{pixel})$

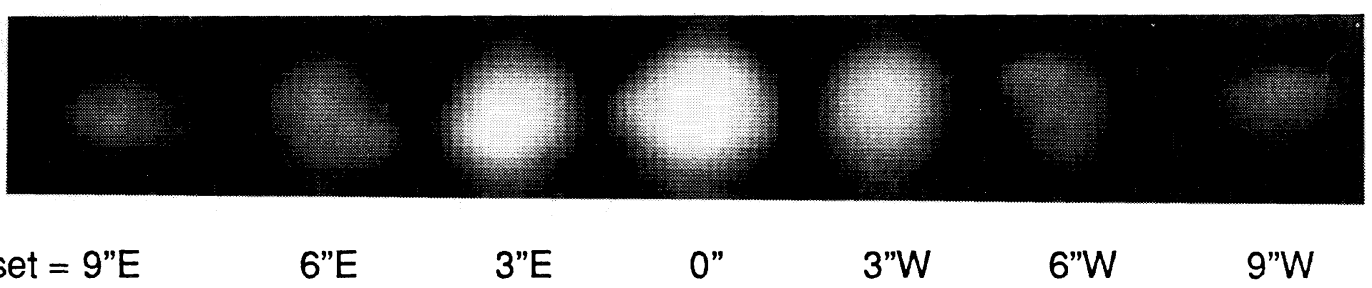

slit offset $=9 " E$

6"E

3"E

O

3"W

$6 W$

g'W

FIG. 2. (continued)

Icke et al. (see page 463) 


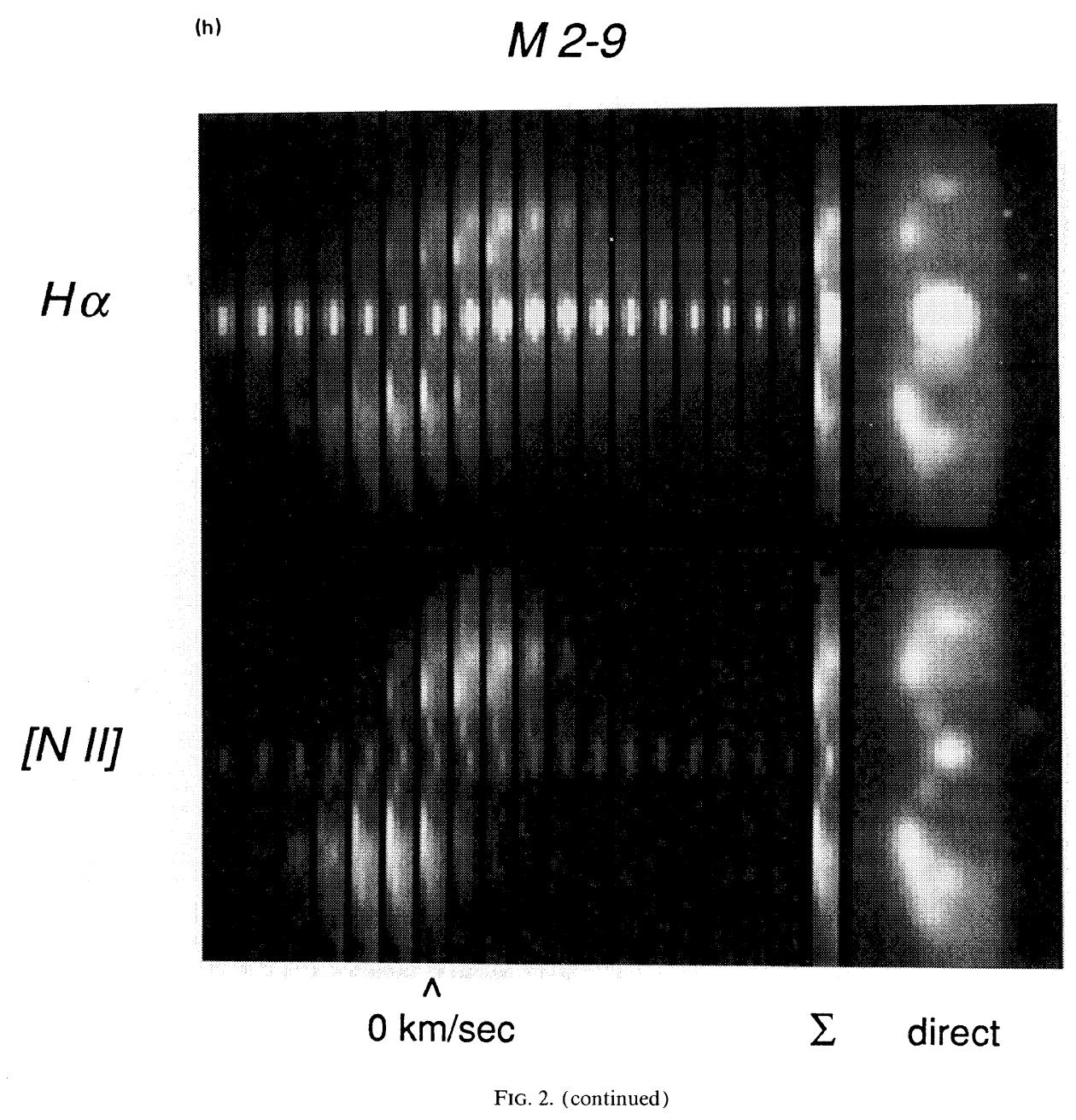

Icke et al. (see page 463) 
NGC 2440
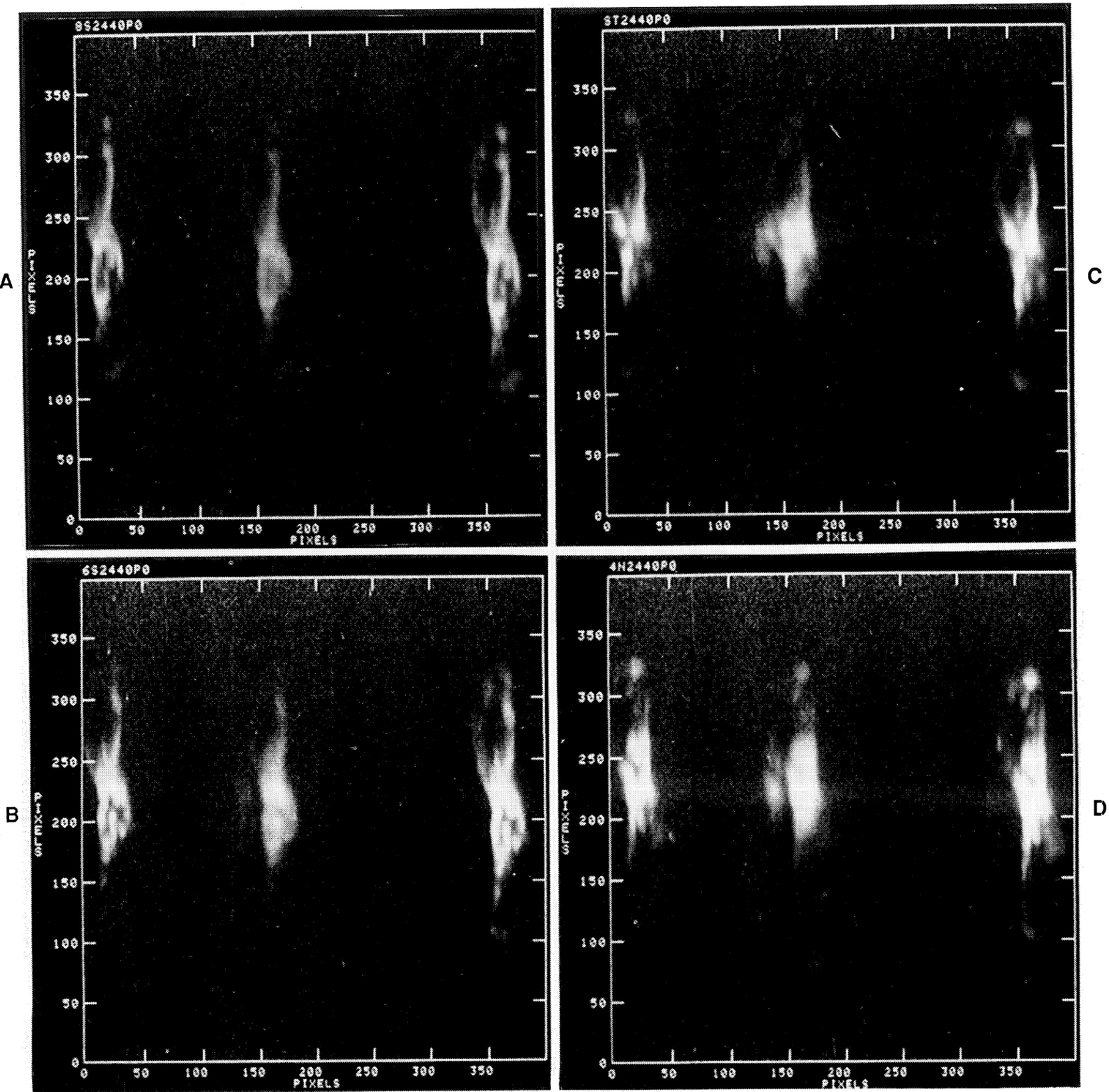

FIG. 10. Line shapes of the planetary nebula NGC 2440. Central line is $\mathrm{H} \alpha$, to the right is [N II]. (a) with the spectrograph slit 8" S of the star, (b) 6" S, (c) slit on the star, (d) slit 4" N. Notice the hourglass shape of the lines, especially [N II], which is less thermally broadened than H $\alpha$. Compare with the theoretical images of Fig. 6.

Icke et al. (see page 473 ) 
NGC 2346

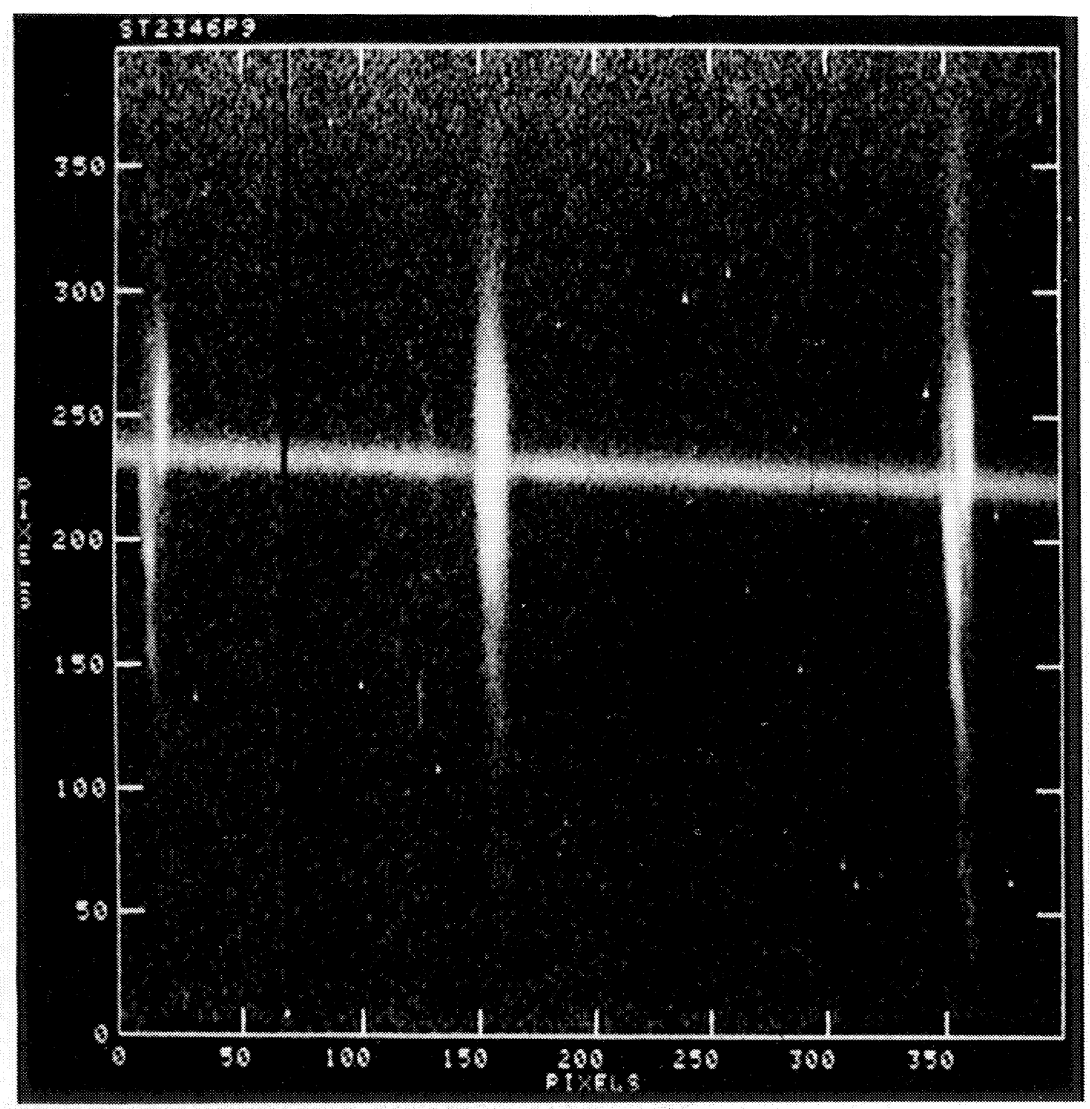

FIG. 11. Line shapes of the planetary nebula NGC 2346. Central line is $\mathrm{H} \alpha$, to the right is [N II]. The spectrograph slit crosses the central star of the nebula. Notice the backward slant of the lines, especially the less thermally broadened [ $\mathrm{N}$ II], showing the "false rotation" phenomenon. Compare with the theoretical images of Fig. 6.

Icke et al. (see page 473) 